\title{
Processing arctic eddy-flux data using a simple carbon-exchange model embedded in the ensemble Kalman filter
}

\author{
Edward B. Rastetter, ${ }^{1,7}$ Mathew Williams, ${ }^{2}$ Kevin L. Griffin, ${ }^{3}$ Bonnie L. Kwiatkowski, ${ }^{1}$ \\ Gabrielle Tomasky, ${ }^{1}$ Mark J. Potosnak, ${ }^{4}$ Paul C. Stoy ${ }^{2,8}$ Gaius R. Shaver, ${ }^{1}$ Marc Stieglitz, ${ }^{5}$ \\ John E. Hobbie, ${ }^{1}$ and George W. Kling ${ }^{6}$ \\ ${ }^{1}$ The Ecosystems Center, Marine Biological Laboratory, 7 MBL Street, Woods Hole, Massachusetts 02543-1015 USA \\ ${ }^{2}$ School of GeoSciences, University of Edinburgh, Crew Building, The King's Buildings, West Mains Road, \\ Edinburgh EH9 $3 J$ N Scotland, United Kingdom \\ ${ }^{3}$ Lamont-Doherty Earth Observatory, Columbia University, 61 Route $9 \mathrm{~W}$, Palisades, New York 10964-8000 USA \\ ${ }^{4}$ Environmental Science Program, DePaul University, 203 F McGowan South, 1110 W. Belden Avenue, \\ Chicago, Illinois 60614-2245 USA \\ ${ }^{5}$ School of Civil and Environmental Engineering, Georgia Institute of Technology, Room 101, Daniel Environmental \\ Engineering Laboratory, 200 Bobby Dodd Way, Atlanta, Georgia 30332-0373 USA \\ ${ }^{6}$ Department of Ecology and Evolutionary Biology, University of Michigan, 2019 Kraus Natural Science Building, \\ 830 North University, Ann Arbor, Michigan 48109-1048 USA
}

\begin{abstract}
Continuous time-series estimates of net ecosystem carbon exchange (NEE) are routinely made using eddy covariance techniques. Identifying and compensating for errors in the NEE time series can be automated using a signal processing filter like the ensemble Kalman filter (EnKF). The EnKF compares each measurement in the time series to a model prediction and updates the NEE estimate by weighting the measurement and model prediction relative to a specified measurement error estimate and an estimate of the model-prediction error that is continuously updated based on model predictions of earlier measurements in the time series. Because of the covariance among model variables, the EnKF can also update estimates of variables for which there is no direct measurement. The resulting estimates evolve through time, enabling the EnKF to be used to estimate dynamic variables like changes in leaf phenology. The evolving estimates can also serve as a means to test the embedded model and reconcile persistent deviations between observations and model predictions.

We embedded a simple arctic NEE model into the EnKF and filtered data from an eddy covariance tower located in tussock tundra on the northern foothills of the Brooks Range in northern Alaska, USA. The model predicts NEE based only on leaf area, irradiance, and temperature and has been well corroborated for all the major vegetation types in the Low Arctic using chamber-based data. This is the first application of the model to eddy covariance data.

We modified the EnKF by adding an adaptive noise estimator that provides a feedback between persistent model data deviations and the noise added to the ensemble of Monte Carlo simulations in the EnKF. We also ran the EnKF with both a specified leaf-area trajectory and with the EnKF sequentially recalibrating leaf-area estimates to compensate for persistent model-data deviations. When used together, adaptive noise estimation and sequential recalibration substantially improved filter performance, but it did not improve performance when used individually.

The EnKF estimates of leaf area followed the expected springtime canopy phenology. However, there were also diel fluctuations in the leaf-area estimates; these are a clear indication of a model deficiency possibly related to vapor pressure effects on canopy conductance.
\end{abstract}

Key words: Alaska, USA; data assimilation; ecosystem carbon balance; ecosystem models; eddy covariance; Kalman filter; net ecosystem carbon exchange.

\section{INTRODUCTION}

The development of eddy covariance techniques has revolutionized the study of ecosystem carbon budgets

Manuscript received 18 May 2009; revised 13 October 2009; accepted 21 October 2009. Corresponding Editor: D. S. Schimel.

${ }^{7}$ E-mail: erastett@mbl.edu

${ }^{8}$ Present address: Department of Land Resources and Environmental Sciences, Montana State University, 334 Leon Johnson Hall, Bozeman, Montana 59717 USA. (e.g., Wofsy et al. 1993, Anthoni et al. 1999, Running et al. 1999, Ehman et al. 2002, Falge et al. 2002a, b, Law et al. 2002, 2003, Baldocchi 2003, Sacks et al. 2006). The long, high-frequency eddy covariance time-series data give a unique, nearly continuous, and nondestructive measure of net ecosystem carbon exchange (NEE) impossible to attain by other methods. These data have also proven to be very valuable to develop, constrain, and test models (e.g., Williams et al. 1996, 2005, Gove and Hollinger 2006). Through the use of inverse modeling, they are a useful way to estimate properties of the surrounding 
TABLE 1. Ensemble Kalman filter (EnKF) components.

\begin{tabular}{ll}
\hline \hline Component of filter, equation & Representation \\
\hline Predict (forecast) & predicted state \\
$\mathbf{x}_{* i(t \mid t-1)}=f\left(\mathbf{x}_{i(t-1 \mid t-1)}, \mathbf{u}_{t}\right)$ & corrupted state estimate \\
$\mathbf{x}_{i(t \mid t-1)}=\mathbf{x}_{* i(t \mid t-1)}+N\left(\mathbf{Q}_{t}\right)$ & ensemble deviations \\
$d_{i t}=\mathbf{x}_{i(t \mid t-1)}-\frac{1}{n} \sum_{i=1}^{n} \mathbf{x}_{i(t \mid t-1)}$ & estimate covariance \\
$\mathbf{P}_{t}=\frac{1}{n} \sum_{i=1}^{n}\left(d_{i t} d_{i t}^{\top}\right)$ & \\
Correct $($ analysis) & innovations \\
$\mathbf{y}_{i t}=\mathbf{z}_{t}-\mathbf{H}_{t} \mathbf{x}_{i(t \mid t-1)}+N\left(\mathbf{\Psi}_{t}\right)$ & innovations covariance \\
$\mathbf{S}_{t}=\frac{1}{n} \sum_{i=1}^{n}\left(\mathbf{y}_{i t} \mathbf{y}_{i t}^{\top}\right)$ & Kalman gain \\
$\mathbf{K}_{t}=\mathbf{P}_{t} \mathbf{H}_{t}^{\top} \mathbf{S}_{t}^{-1}$ & updated state \\
$\mathbf{x}_{i(t \mid t)}=\mathbf{x}_{i(t \mid t-1)}+\mathbf{K}_{t} \mathbf{y}_{i t}$ & \\
\hline Note See Table 4 for symbols and definitions.
\end{tabular}

vegetation that would otherwise be difficult to measure (e.g., canopy light adsorption; Hanan et al. 2002).

As with any measurement, errors in eddy covariance data can arise from several sources. It is therefore important to identify and compensate for these errors. A typical first step in the analysis of any type of data is to plot and examine the data so that obvious errors can be identified and removed before further analysis. For timeseries data, the analogous procedure can be automated through the use of a signal processing filter like the ensemble Kalman filter (EnKF; Evensen 2003). In the filter, potential errors are identified by comparing each measurement in the time series to the predictions of a model (e.g., a NEE model). Instead of removing the suspect data, the filter replaces the measurements with a weighted mean of the measurement and the model prediction; the relative weighting is based on a specified estimate of measurement error and an estimate of the model-prediction error that is continuously updated based on how well the model predicted earlier measurements in the time series.

The EnKF can also be used for data assimilation (Wang et al. 2000). Because of the covariance among variables in the model, measurements assimilated through the EnKF can be used to constrain estimates of variables for which there are no direct measurements. Unlike many data assimilation approaches (e.g., Wu et al. 2009), variable estimates in the EnKF are updated with each measurement in the time series. The estimates therefore evolve through time, thereby providing a means to estimate dynamic variables like phenological changes in leaf area. In addition, the sequential updating of estimates continuously recalibrates the model to current conditions, thereby compensating for model inadequacies. The time trajectory of estimated variables provides a means to identify those inadequacies and thereby test the model.
Here we present a signal processing filter for eddy covariance data based on a model of arctic NEE (Shaver et al. 2007) embedded in an EnKF. The model was developed from chamber-based estimates of NEE, but has never before been applied to eddy covariance data. Our application of the model and the EnKF has three goals: (1) signal processing to assess the capabilities of the linked model-EnKF to filter random noise from the eddy covariance data stream, (2) data assimilation to use the linked model EnKF to estimate the phenological changes in leaf area around the eddy covariance tower, and (3) model testing to test the embedded NEE model against eddy covariance data using the EnKF. Although the NEE model used here is strictly applicable only to low arctic ecosystems, models for other ecosystems could be used within the same signal processing scheme.

\section{KaLman Filtering}

The Kalman filter (KF; Brown 1983, Young 1984) is a signal processing algorithm that can be used to filter noise-corrupted time series based on sequential, maximum-likelihood fits to an embedded model. The approach is Bayesian, requiring both a prediction (or forecasting) step and a correction (or analysis) step. In the prediction step, the model is used to estimate a future state of the system (e.g., net ecosystem carbon exchange [NEE]) and to project a priori model uncertainty forward in time. In the correction step, the model prediction is compared to measurements and adjusted based on an assessment of the projected model uncertainty relative to the measurement error. Adjustments can also be made to unobserved variables in the model based on their covariance with the observed variables. In this way, confidence in the model is used to reduce noise in the measurements, and the measurements are continuously used to recalibrate both observed and unobserved components of the model and to quantify model uncertainty over time. The original KF could only be coupled to a linear model (Brown 1983, Young 1984). However, subsequent versions have extended the application to nonlinear models (e.g., the extended KF [Brown 1983, Cosby and Hornberger 1984, Young 1984]; the unscented KF [Gove and Hollinger 2006]; and the ensemble KF [EnKF; Evensen 2003, Williams et al. 2005, Chen et al. 2008]).

The EnKF is a Monte Carlo based version of the KF that is particularly straightforward to use with nonlinear models. Before applying the EnKF (Table 1), a Monte Carlo procedure is used to generate a random ensemble of initial model state vectors $\left(\mathbf{x}_{* i(0 \mid 0)}\right)$. The EnKF then uses a model $(f)$ to predict the future system states $\left(\mathbf{x}_{* i(t \mid t-1)}\right)$ for the ensemble based on the ensemble of past states $\left(\mathbf{x}_{i(t-1 \mid t-1)}\right)$ and a vector of external drivers $\left(\mathbf{u}_{t}\right)$. The Monte Carlo procedure is then used to corrupt each member of this ensemble of predictions with noise $\left(N\left(\mathbf{Q}_{t}\right)\right)$ to reflect model uncertainty. As with most applications, we will assume that this noise is zero mean, Gaussian, and that the noise is independent among state variables. The uncertainty propagated forward from previous model 
steps plus the uncertainty associated with the current model step $\left(N\left(\mathbf{Q}_{t}\right)\right)$ is quantified in an estimate covariance matrix $\left(\mathbf{P}_{t}\right)$, which is approximated by the covariance matrix for the ensemble of Kalman state vectors.

To compare each member of the ensemble to observations, the modeled variables for which there are observations are first extracted from the Kalman state vector using an observation matrix $\left(\mathbf{H}_{t}\right)$. An ensemble of innovations $\left(\mathbf{y}_{i t}\right)$ is then generated by subtracting the model predictions from the observations and adding noise $\left(N\left(\boldsymbol{\Psi}_{t}\right)\right)$ to reflect uncertainty associated with the measurements (again assumed zero-mean, Gaussian, and independent among observation variables). The total model plus measurement uncertainty is quantified in the covariance matrix for the ensemble of innovations $\left(\mathbf{S}_{t}\right)$ assuming that the model is unbiased (i.e., assuming the mean of the $\mathbf{y}_{i t}$ over the ensemble is zero). A correction matrix, the Kalman gain $\left(\mathbf{K}_{t}\right)$, is then calculated from the estimate covariance $\left(\mathbf{P}_{t}\right)$ and the innovations covariance $\left(\mathbf{S}_{t}\right)$; in essence, the Kalman gain quantifies the fraction of the total uncertainty that can be attributed to the model. Finally, the ensemble of estimated state vectors is corrected using the Kalman gain and the ensemble of innovations. This correction is imposed on both the observed and unobserved components of the state vector; corrections on the unobserved components are based on their covariance with the observed components as quantified in the estimate covariance matrix $\left(\mathbf{P}_{t}\right)$.

If uncertainty in the model is high relative to the total uncertainty ( $\mathbf{K}_{t}$ near 1), then a large correction is imposed and the ensemble of corrected state vectors will collapse close to the observation. If the uncertainty in the model is low relative to the total uncertainty ( $\mathbf{K}_{t}$ near 0$)$, then only a small correction is imposed and the spread in the values of the ensemble of state vectors remains relatively unchanged. The mean of the corrected estimates of the ensemble is the EnKF output and represents the best estimate of the true value of the Kalman state vector $\mathbf{x}$. The spread among the ensemble of corrected estimates quantifies the confidence in that output.

\section{Compensating for Persistent Deviations And Model Testing}

The model embedded in the ensemble Kalman filter $($ EnKF) is at best only an approximation of the real system it is intended to represent. It is therefore possible that important processes have been either misrepresented or unspecified in the model. These model deficiencies result in deviations between predictions and observations that can be usefully partitioned into two categories:

1) Model deficiencies resulting in nonperiodic deviations that have durations that are shorter than twice the sampling interval in the time-series data and

2) Model deficiencies resulting in periodic deviations or deviations that are persistent for more than twice the sampling interval in the time-series data.
Deviations of the first type might arise, for example, in eddy covariance data during the transition from a stable to turbulent boundary layer (Papale et al. 2006) or as the result of rapid transitions in the nighttime radiation budget with the passage of clouds (Cava et al. 2004). Without some other source of information to resolve the underlying mechanisms, there is no way of knowing from the NEE time series itself if deviations of the first type are the result of a flaw in the model or random measurement errors. We therefore treat such deviations as random errors, fully recognizing that they might represent real processes that cannot be resolved in our data.

Because of the temporal autocorrelation in deviations of the second type, they are a clear indication of either a deficiency in the model (Lin and Beck 2007) or a comparable drift or bias in the instrumentation used to measure the input $\left(\mathbf{u}_{t}\right)$ or observation $\left(\mathbf{z}_{t}\right)$ time series (e.g., Burba et al. 2008). Again, there is no way to distinguish between these two possibilities based on the time-series data alone. We will assume that any such deviations are the result of a deficiency in the model but include the possibility of instrument drift in the postfiltering analysis of the results. More importantly, the autocorrelation in these deviations makes it possible to compensate (at least partially) for them in a signal processing context (i.e., data noise filtering) and provides diagnostic insights into why and how the model failed in a model-testing context.

The objective of signal processing is to filter random noise from the observation $\left(\mathbf{z}_{t}\right)$ time series. Deviations of the second type listed are clearly not random and ideally should not be filtered from the time series. Because of their autocorrelation, they can be compensated for by sequentially recalibrating the model to fit the observations. If the deviations are autocorrelated, then a recalibration to the current measurement should decrease the deviations for the next measurement (we assume positive autocorrelations to avoid the problems inherent with negative autocorrelations). This recalibration will automatically be accomplished through the correction step of the EnKF if the appropriate calibration variable is included as an unobserved component of the Kalman state vector (x). For this automatic recalibration to work, the calibration variable must have two properties: (1) it must have a direct influence on the value of the observed variable with the autocorrelated deviations, and (2) it must itself be autocorrelated so that the effects of recalibration are passed from time step to time step. If such a variable does not already exist in the Kalman state vector, then the vector can be augmented with a parameter that directly influences the observed variable. To assure autocorrelation for this augmented parameter, its value is set to its previous value by the model embedded in the EnKF $(f)$. Its value is corrupted by the Monte Carlo procedure following the prediction step and is adjusted (i.e., recalibrated) based on its covariance with the 
observed variables in the correction step (quantified in $\left.\mathbf{P}_{t}\right)$.

This correction step adjusts the variable toward a value that is more consistent with the observations (i.e., recalibrates the model). However, a single random deviation in the observations (i.e., random measurement errors or deviations of the first type listed above) results in a decrease in the Kalman gain for the current step (elements of $\mathbf{S}_{t}$ are larger but $\mathbf{P}_{t}$ is unaffected) and therefore only results in small adjustments to the variable in the current step. The uncertainty is therefore propagated forward to the model predictions in the following step. If the deviations are persistent (i.e., the second type of deviations), then the model uncertainty builds through time and the variable adjustments increase until the filter converges on a new calibration of the model.

Sequential recalibration of the unobserved components of the Kalman state vector can also serve as a diagnostic tool for assessing the cause of the second type of deviation (Beck and Young 1976, Cosby et al. 1984) and thereby help identify potential problems with the embedded model. Through the sequential recalibration, the autocorrelated deviations in the predictions are transposed onto the unobserved components of the Kalman state vector (although corrupted to account for model uncertainty by the Monte Carlo procedure). Thus, the sequential recalibration is a means of segregating the autocorrelated, type-two deviations from the random, type-one deviations. In addition, for more complex models and multiple observation variables, the transposed deviations are isolated to specific process variables or parameters through their covariance with the observed variables (as quantified in $\mathbf{P}_{t}$ ). Thus the EnKF can help identify which part of the model is deficient.

\section{Adaptive Noise Estimation}

One of the most difficult and subjective aspects of applying the Kalman filter is quantifying the uncertainty associated with the current model step (specifying $\mathbf{Q}_{t}$ in Table 1). This difficulty is particularly acute when the filter compensates for deviations caused by misrepresented or unspecified processes in the model. As a consequence, the appropriate values of the elements of $\mathbf{Q}_{t}$ can change, being relatively small when the misrepresented processes have little effect on the system behavior, but large when they cause major shifts in the system state. Setting the values in $\mathbf{Q}_{t}$ too small will cause the filter to respond and converge slowly after a shift in system state (Fig. 1A). Setting the values in $\mathbf{Q}_{t}$ too large will allow the filter to converge rapidly after a state shift, but will result in poor filtering of the observations because of unnecessarily large model uncertainty once the filter has converged on the new state (Fig. 1B).

To alleviate this problem, Jazwinski (1998; see also Vallino 1985) proposed an adaptive noise estimation scheme in which $\mathbf{Q}_{t}$ adapts to changes in the magnitude of the innovations $\left(\mathbf{y}_{i t}\right)$. Thus, if the innovations are persistently large because of a model bias, the values in $\mathbf{Q}_{t}$ increase, allowing the filter to converge on the observations more rapidly (Fig. 2). Once the filter has converged on the observations, the values in $\mathbf{Q}_{t}$ again decrease to a magnitude consistent with the variation in the innovations (Fig. 3). We adapted Jazwinski's scheme to the EnKF (Table 2).

The essence of the Jazwinski (1998) approach is based on the observation that if the model is unbiased, then the innovations covariance is the sum of three sources of uncertainty: the measurement error, the error propagated forward in time by the model, and the current model error, given as $\mathbf{S}_{t}=\boldsymbol{\Psi}_{t}+\mathbf{H}_{t} \mathbf{P}_{*} \mathbf{H}_{t}^{\top}+\mathbf{H}_{t} \mathbf{Q}_{t} \mathbf{H}_{t}^{\top}$, where $\mathbf{P}_{* t}$ is the covariance matrix of the ensemble of predicted Kalman state vectors $\left(\mathbf{x}_{* i(t \mid t-1)}\right)$ before they are corrupted by $\boldsymbol{N}\left(\mathbf{Q}_{t}\right)$ (Table 2) and $\mathbf{P}_{*}$ and $\mathbf{Q}_{t}$ are pre- and post multiplied by the measurement matrix and its transpose $\left(\mathbf{H}_{t}\right.$ and $\left.\mathbf{H}_{t}^{\top}\right)$ to extract only those components associated with the measured variables. An estimate of $\mathbf{H}_{t} \mathbf{Q}_{t} \mathbf{H}_{t}^{\top}$ can then be calculated by difference. However, this residual must then be distributed among the observed and unobserved state variables by pre- and post-multiplying by an error distribution matrix and its transpose $\left(\boldsymbol{\Gamma}_{t}\right.$ and $\left.\boldsymbol{\Gamma}_{t}^{\top}\right)$. Finally, to avoid oversensitivity to random perturbations (Jazwinski 1998), the new estimate of $\mathbf{Q}_{t}$ is averaged with the previous estimate using a weighting factor $(\alpha)$ that regulates how quickly $\mathbf{Q}_{t}$ is allowed to adapt.

To build the error distribution matrix, $\boldsymbol{\Gamma}_{t}$, we assume that the model has been written so that the observations can be compared directly to the corresponding state variables without transformation (Table 2). Thus, the measurement matrix, $\mathbf{H}_{t}$, is composed of only 1's and 0 's; there is only a single 1 per row; and there is at most a single 1 per column. This assumption allows us to build $\Gamma_{t}$ based on a regression of the ensemble of unobserved variables on the ensemble of observed variables using the estimate covariance matrix $\left(\mathbf{P}_{t}\right.$; based on the corrupted state vectors). The partial regression coefficients in the portion of $\boldsymbol{\Gamma}_{t}$ associated with unobserved variables are calculated as $\left(\mathbf{H}_{t} \mathbf{P}_{t} \mathbf{H}_{t}^{\top}\right)^{-1} \mathbf{H}_{t} \mathbf{P}_{t}\left(\mathbf{I}-\mathbf{H}_{t}^{\top} \mathbf{H}_{t}\right)$, which will distribute uncertainty in the observed variables onto the unobserved variables, where $\mathbf{I}$ is the identity matrix. The uncertainty in the observed variables is accounted for by adding the measurement matrix $\left(\mathbf{H}_{t}\right)$ to fill in the portion of $\boldsymbol{\Gamma}_{t}$ associated with the observed variables.

By accounting for the uncertainty both directly through the observed variables and indirectly through the regression of the unobserved variables on the observed variables, we have accounted for the uncertainty twice (if the regressions were perfect). That is, the noise imposed on the unobserved variables [through $N\left(\mathbf{Q}_{t}\right)$ ] will be propagated through the model (by the function $f$ ) and added to the noise already imposed directly on the observed variables. To correct for this 

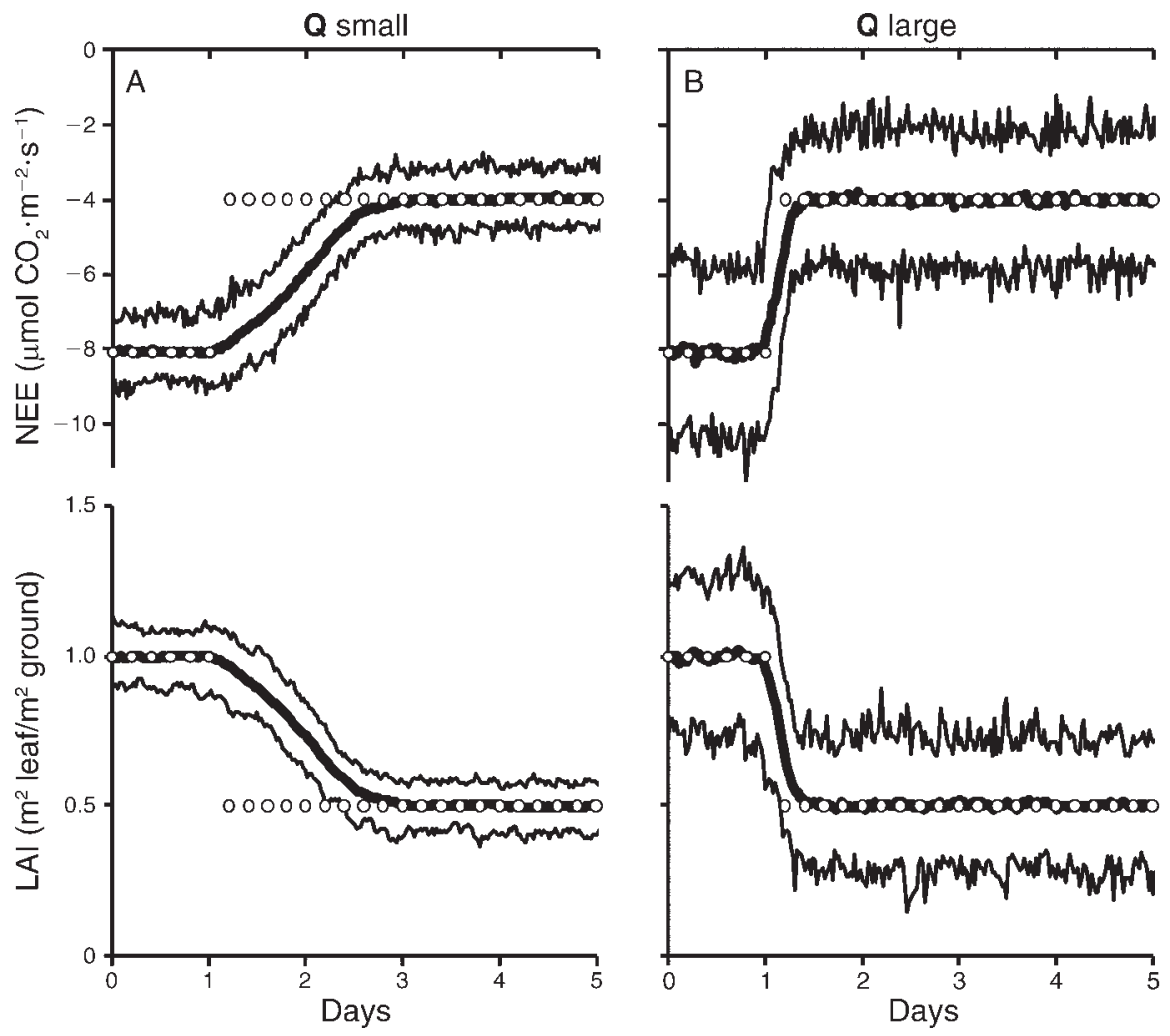

FIG. 1. Performance of the ensemble Kalman filter (EnKF) with large and small model noise estimates. The PLIRTLE model (Eq. 1; Shaver et al. 2007) was used to simulate net ecosystem carbon exchange (NEE) under constant light and temperature, but with a major defoliation event on day 1 (open circles). PLIRTLE was then embedded in the EnKF with the leaf area index (LAI) included as an estimated, but unobserved, variable in the Kalman state vector. When the noise corruption $\left[N\left(\mathbf{Q}_{t}\right)\right.$; see Table 1] is $\sim 1 \%$ of NEE and LAI (Q small; panel A), the mean ensemble estimates of NEE and LAI (thick black lines) slowly converge on the true values following the defoliation event. However, once the EnKF converges on the new condition, the ensemble of model estimates is tightly constrained (thin black lines enclose the range of the 100 simulations in the ensemble). When the noise corruption is $\sim 10 \%$ ( $\mathbf{Q}$ large; panel B), the mean ensemble estimates converge quickly, but the ensemble of model estimates is poorly constrained.

double accounting, we add a weighting factor $(0<\beta<$ 1) that distributes the uncertainty between the unobserved and observed components of the Kalman state vector. If $\beta$ is small, most of the uncertainty is distributed to the unobserved state variables. If $\beta$ is large, most of the uncertainty is distributed to the observed variables (Fig. 2). The best value for $\beta$ will depend upon the model, the observations, and the measurement frequency, but can be found through an analysis of the variances in the filter. We present this analysis in the Results section.

In our application we have only two components to the Kalman state vector, thus $\beta$ allows us to partition error specifically between the two components. With a higher dimension state vector, it might be possible to customize the error-distribution matrix to have a more targeted distribution of uncertainty.

\section{The PLIRTLE NEP Model}

We embedded the PLIRTLE model of Shaver et al. (2007) into the EnKF [the name PLIRTLE derives from the functional representation $P(L, I)-R(T, L)]$ :

$$
\begin{gathered}
F_{\mathrm{C}}=-[P(L, I)-R(T, L)] \\
R(T, L)=R_{0}+R_{\mathrm{L}} L e^{\phi T} \\
P(L, I)=\frac{P_{\max }}{k} \ln \left(\frac{P_{\max }+E_{0} I}{P_{\max }+E_{0} I e^{-k L}}\right)
\end{gathered}
$$

where $F_{\mathrm{C}}, P$, and $R$ are net ecosystem carbon exchange (NEE), photosynthesis, and ecosystem respiration (all in $\mu \mathrm{mol} \mathrm{CO}_{2} \cdot\left[\mathrm{m}^{2} \text { ground }\right]^{-1} \cdot \mathrm{s}^{-1}$ ), $L$ is the leaf area index $\left(\mathrm{m}^{2}\right.$ leaf $/ \mathrm{m}^{2}$ ground $), T$ is air temperature $\left({ }^{\circ} \mathrm{C}\right), \quad I$ is photosynthetic proton flux density (PPFD; $\mu \mathrm{mol}$ photons $\cdot\left[\mathrm{m}^{2} \text { ground }\right]^{-1} \cdot \mathrm{s}^{-1}$ ), $R_{0}$ and $R_{\mathrm{L}}$ are respiration parameters $\left(\mu \mathrm{mol} \quad \mathrm{CO}_{2} \cdot\left[\mathrm{m}^{2}\right.\right.$ ground $]^{-1} \cdot \mathrm{s}^{-1}$ and $\mu \mathrm{mol} \mathrm{CO}_{2} \cdot\left[\mathrm{m}^{2} \text { leaf }\right]^{-1} \cdot \mathrm{s}^{-1}$, respectively), $\phi$ is the temperature response coefficient for respiration $\left({ }^{\circ} \mathrm{C}^{-1} ; \mathrm{Q}_{10}=\mathrm{e}^{10 \phi}\right), P_{\max }$ is the maximum foliar photosynthetic rate $\left(\mu \mathrm{mol} \mathrm{CO} \mathrm{CO}_{2} \cdot\left[\mathrm{m}^{2} \text { leaf }\right]^{-1} \cdot \mathrm{s}^{-1}\right)$, $E_{0}$ is the quantum yield ( $\mu \mathrm{mol} \mathrm{CO}_{2} / \mu \mathrm{mol}$ photons), 

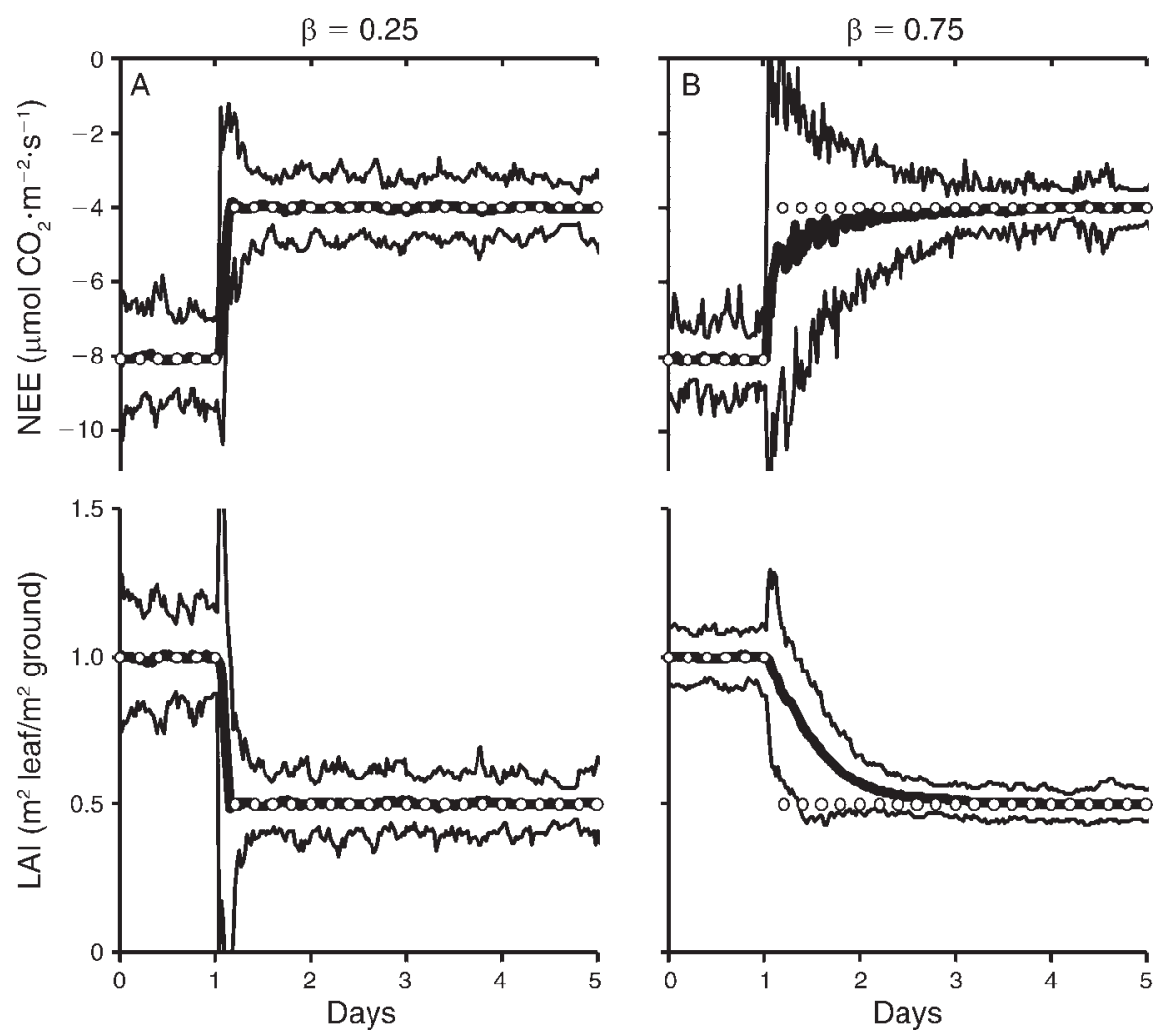

FIG. 2. Performance of the EnKF with adaptive model noise estimates. Simulations are as in Fig. 1, but with an adaptive algorithm to allow changes in $\mathbf{Q}_{t}$. Two values of the weighting factor $(\beta)$ in the adaptive noise feedback were used. (A) When $\beta$ is small, most of the uncertainty is distributed to the LAI (the unobserved variable). The noise corruption on the LAI therefore increases following the defoliation event, allowing the filter to "explore" a wide range of LAI values, and the filter converges quickly. (B) When $\beta$ is large, most of the uncertainty is distributed to NEE (the observed variable). The noise corruption on the LAI therefore increases only slightly following the defoliation event, and the filter converges slowly.

and $k$ is Beer's light extinction coefficient $\left(\mathrm{m}^{2}\right.$ ground/ $\mathrm{m}^{2}$ leaf).

The most remarkable characteristics of this model are (1) only PPFD, air temperature, and leaf area are required to reliably predict NEE for all the major vegetation types in the low arctic $\left(r^{2}>0.77\right)$ and (2) the same values for the six parameters apply to all major arctic vegetation types in both northern Sweden and the North Slope of Alaska (Shaver et al. 2007; Table 3). Shaver et al. (2007) derived this model from many chamber-based flux measurements (1410 individual flux estimates); this is the first application of the model to eddy covariance data. We assumed that the eddy covariance NEE estimate above the canopy is approximately equal to the negative of net ecosystem production (NEP); i.e., we assumed 0 lateral carbon exchange and negligible recycling of $\mathrm{C}$ respired below the eddy covariance sensor (Goulden et al. 1997).

We had no direct estimate of the leaf area in the tower footprint and therefore had to include it as a predicted variable in the model. With this modification to the model, we were able to use the EnKF to estimate leaf area based on the assimilated eddy covariance data; this application of the EnKF is analogous to the Hanan et al. (2002) inverse modeling estimates of canopy light adsorption.

Because the data we used were for early spring when leaves were expanding, we expected leaf area to increase steadily through the measurement period and the rate of increase to be proportional to temperature (approximately equivalent to leaf area increasing in proportion to the degree-day sum):

$$
\frac{d L}{d t}=a T
$$

However, we had no independent way to estimate the rate constant $\left(a\right.$ in $\left.\mathrm{m}^{2} \cdot \mathrm{m}^{-2} \cdot{ }^{\circ} \mathrm{C}^{-1}\right)$ and there is not enough information in the NEE time series to estimate both $L$ and $a$ in an augmented state vector. Instead, because we expected the rate of leaf area increase to be slow relative to the half-hour sampling interval of the eddy covariance data, we assumed no change in leaf area in the model and let the EnKF update leaf area estimates through the correction step:

$$
\frac{d L}{d t}=0 .
$$

This estimation of leaf area provides the only autocor- 

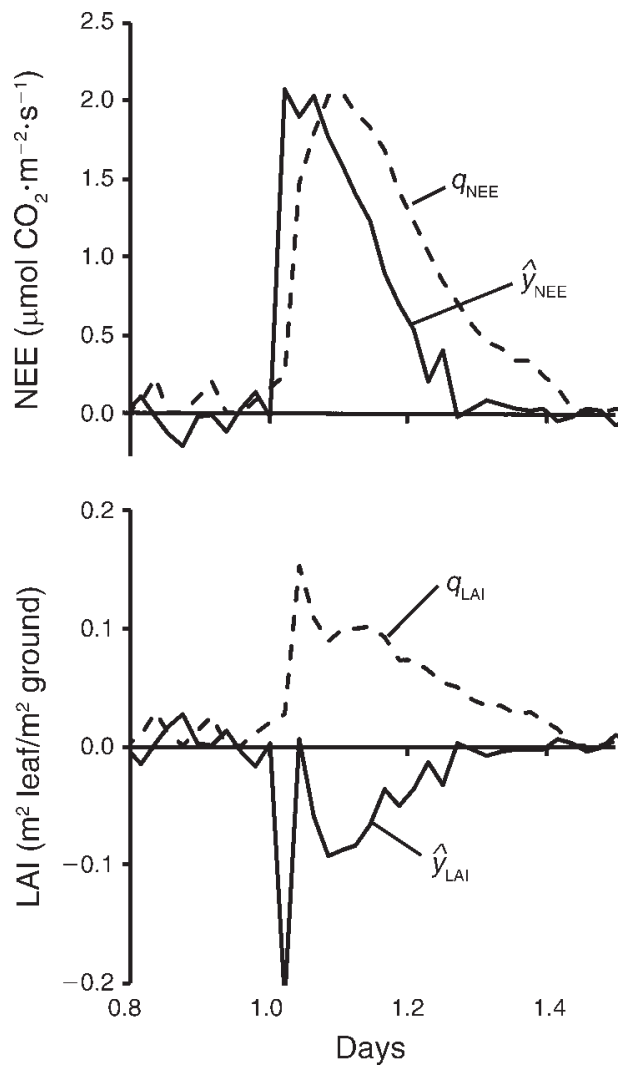

FIG. 3. Coupled dynamics of the adaptive noise estimates and EnKF innovations. The EnKF innovations $\left(\mathbf{y}_{i t}\right)$ were averaged over the ensemble and distributed over the observed and unobserved elements of the Kalman state vector through the relationship $\hat{y}_{t}=\boldsymbol{\Gamma}_{t} \bar{y}_{\mathrm{t}}$, where the bar indicates averaging over the ensemble and $\beta$ in the calculation of $\boldsymbol{\Gamma}_{t}$ was set to 0.55 . The square roots of the elements in $\mathbf{Q}_{t}$ are $q_{\mathrm{NEE}}$ and $q_{\mathrm{LAI}}$, associated, respectively, with NEE and LAI. A persistent deviation of the mean innovations from 0 feeds back through the adaptive noise estimation (Table 2) to increase the elements in the noise matrix $\mathbf{Q}_{t}$. When the innovations again approach 0 , the elements in $\mathbf{Q}_{t}$ also decrease in response.

relation in the model and will not only evolve with the expected advance in springtime phenology, it will also subsume any autocorrelated deviations associated with deficiencies in the PLIRTLE model. These autocorrelated deviations provide the basis for testing the PLIRTLE model and for diagnosing its deficiencies.

\section{ANALYSIS}

We embedded the PLIRTLE model into the EnKF and applied it to eddy covariance time series from the North Slope of the Brooks Range in Alaska, USA. The eddy covariance tower is located in moist tussock tundra in the Imnavait Creek watershed $\sim 12 \mathrm{~km}$ east of the Toolik Lake Arctic Long-Term Ecological Research site at an elevation of $930 \mathrm{~m}$ above sea level. This site was included in the chamber-based flux measurements used to derive the PLIRTLE model and a more detailed description of the site can be found in Shaver et al.
(2007). Data were for a period from 11 to 24 June 2004, which is just after snow melt and during the period of leaf expansion for most of the plant species.

Eddy covariance data were collected with an openpath $\mathrm{CO}_{2} / \mathrm{H}_{2} \mathrm{O}$ gas analyzer (LI-7500, LI-COR Biosciences, Lincoln, Nebraska, USA) and sonic anemometer (CSAT3, Campbell Scientific, Logan, Utah, USA) located $\sim 2 \mathrm{~m}$ above the land surface. Data from these instruments were collected with a CR5000 datalogger (Campbell Scientific) using a digital interface at $20 \mathrm{~Hz}$. The site is located on the slope of the Imnavait Creek watershed with an inclination of $\sim 6^{\circ}$. To remove the bias due to nonzero vertical winds that follow the slope, wind data were rotated using the planar fit method (Wilczak et al. 2001, Turnipseed et al. 2003). This method considers the entire wind field data set and determines a single set of two rotation angles applied to all data. After rotation, the covariance between the $\mathrm{CO}_{2}$ concentration and the vertical wind over 30 min periods was corrected for the effects of air density fluctuations owing to sensible and latent heat fluxes (Webb et al. 1980). All data analysis was performed using R ( R Development Core Team 2006). We selected two weeks of data that were relatively gap free. Gaps in temperature and PPFD data were filled using data from the nearby $(\sim 1 \mathrm{~km})$ North Slope, Imnavait Basin site of Kane and Hinzman (2004). Analysis confirmed a high correlation between the two sites for both temperature $\left(r^{2}=0.91, \quad F_{1441,1442}=15217, P<0.001\right)$ and photosynthetic proton flux density (PPFD; $r^{2}=0.87$, $\left.F_{1127,1128}=7313, P<0.001\right)$. If data from Kane and Hinzman (2004) were unavailable to fill particular gaps, we interpolated between adjacent points. For gaps in the NEE data, we allowed the EnKF to do its own filling by simply turning off the correction step in the EnKF when observations were unavailable.

Richardson et al. (2006) estimated eddy covariance measurement error by comparing measurements made during the same half-hourly interval of adjacent days in which the PPFD, temperature, wind speed, and wind direction of the two days were within a narrow range of

TABle 2. Adaptive noise estimation.

\begin{tabular}{rlr}
\hline \hline \multicolumn{1}{c}{ Equation } & \multicolumn{1}{c}{ Representation } \\
\hline $\boldsymbol{\Gamma}_{t}=\left((1-\beta)\left(\mathbf{H}_{t} \mathbf{P}_{t} \mathbf{H}_{t}^{\top}\right)^{-1}\right.$ & error distribution matrix \\
& $\left.\times \mathbf{H} \mathbf{P}_{t}\left(\mathbf{I}-\mathbf{H}_{t}^{\top} \mathbf{H}_{t}\right)+\beta \mathbf{H}_{t}\right)^{\top}$ & \\
$\mathbf{d}_{* i t}=\mathbf{x}_{* i(t \mid t-1)}-\frac{1}{n} \sum_{i=1}^{n} \mathbf{x}_{* i(t \mid t-1)}$ & $\begin{array}{c}\text { uncorrupted ensemble } \\
\text { deviations }\end{array}$ \\
$\mathbf{P}_{* t}=\frac{1}{n} \sum_{i=1}^{n}\left(\mathbf{d}_{* i t} \mathbf{d}_{* i t}^{\top}\right)$ & $\begin{array}{c}\text { uncorrupted estimate } \\
\text { covariance }\end{array}$ \\
$\hat{\mathbf{Q}}_{t}=\boldsymbol{\Gamma}_{t}\left(\mathbf{S}_{t}-\mathbf{H}_{t} \mathbf{P}_{* t} \mathbf{H}_{t}^{\top}-\Psi\right) \boldsymbol{\Gamma}_{t}^{\top}$ & distributed error matrix \\
$\mathbf{Q}_{t+1}=\alpha \mathbf{Q}_{t}+(1-\alpha) \hat{\mathbf{Q}}_{t}$ & time-smoothed error matrix \\
\hline
\end{tabular}

Note: See Table 4 for symbols and definitions. 
TABle 3. Parameter values for the PLIRTLE model.

\begin{tabular}{lc}
\hline \hline \multicolumn{1}{c}{ Parameter } & Value \\
\hline$P_{\max }\left(\mu \mathrm{mol} \mathrm{CO} \mathrm{CO}_{2} \cdot\left[\mathrm{m}^{2} \text { leaf }\right]^{-1} \cdot \mathrm{s}^{-1}\right)$ & 15.8 \\
$E_{0}(\mu \mathrm{mol} \mathrm{CO} 2 / \mu \mathrm{mol}$ photons $)$ & 0.036 \\
$k\left(\mathrm{~m}^{2}\right.$ ground $/ \mathrm{m}^{2}$ leaf $)$ & 0.5 \\
$R_{0}\left(\mu \mathrm{mol} \mathrm{CO} \mathrm{CO}_{2} \cdot\left[\mathrm{m}^{2} \text { ground }\right]^{-1} \cdot \mathrm{s}^{-1}\right)$ & 0.547 \\
$R_{L}\left(\mu \mathrm{mol} \mathrm{CO} \mathrm{CO}_{2} \cdot\left[\mathrm{m}^{2} \text { leaf }\right]^{-1} \cdot \mathrm{s}^{-1}\right)$ & 0.602 \\
$\phi\left({ }^{\circ} \mathrm{C}^{-1}\right)$ & 0.074 \\
\hline
\end{tabular}

Note: Data are from Shaver et al. 2007: Table 5.

each other. This analysis requires a large volume of data that was not available to us for the Alaska site. Instead we used data from a Swedish tundra site (Fox et al. 2008) that were also used in the development of the PLIRTLE model. This Swedish site has similar vegetation and flux rates as our Alaska site (Shaver et al. 2007). We followed the Richardson et al. (2006) protocols with thresholds for quantifying meteorological similarity on adjacent days taken to be PPFD within 75 $\mu \mathrm{mol} \cdot \mathrm{m}^{-2} \cdot \mathrm{s}^{-1}$, temperature within $2^{\circ} \mathrm{C}$, wind speed within $1 \mathrm{~m} / \mathrm{s}$, and wind direction within $45^{\circ}$. If these conditions were met, differences in the corresponding fluxes were used to estimate error magnitudes. Using this approach, NEE measurement error was found to be a function of flux magnitude (Hollinger and Richardson 2005) that can be described using

$$
\sigma_{\mathrm{NEE}}^{2}= \begin{cases}\left(0.5-0.11 F_{\mathrm{C}}\right)^{2} & \text { if } F_{\mathrm{C}}<0 \\ \left(0.5+0.15 F_{\mathrm{C}}\right)^{2} & \text { if } F_{\mathrm{C}} \geq 0\end{cases}
$$

where $\sigma_{\mathrm{NEE}}^{2}$ is the element on the main diagonal of $\boldsymbol{\Psi}_{t}$ corresponding to NEE.

We maintained all six of the original PLIRTLE parameters as fixed parameters (i.e., not added to an augmented Kalman state vector) with the values reported by Shaver et al. (2007: Table 3). However, we only had time-series data for two of the driver variables, PPFD and air temperature; leaf area estimates were unavailable. We therefore modeled leaf area as described in Eq. 2a. The only variables in the Kalman state vector were $F_{\mathrm{C}}(\mathrm{NEE})$ and $L(\mathrm{LAI}), F_{\mathrm{C}}$ being observed and $L$ unobserved.

We used an ensemble size $(n)$ of 100 . The value of the time-smoothing parameter $(\alpha)$ in the adaptive noise estimation was set to 0.5 to allow for fairly rapid adaptation of $\mathbf{Q}_{t}$. This value of $\alpha$ means that the influence of the current innovations is twice as strong as that for the innovations from the previous half hour, four times as strong as that for the innovations from the half hour prior to that, and so on. We allowed this rapid adaptation in $\mathbf{Q}_{t}$ so that we could detect and account for inadequacies in the model that would manifest on a diel cycle. We examined a range in $\beta$ from 0 to 1 and present the results along with the EnKF analysis of tundra flux assimilation in the next section.

\section{Results And Discussion \\ Error-distribution weighting factor $(\beta)$}

The function of $\beta$ in our adaptive noise estimation is to balance the distribution of Monte Carlo variation imposed on the observed vs. unobserved components of the Kalman state vector. Setting this balance helps optimize the signal processing performance of the filter by sequentially recalibrating the unobserved components of the state vector to help compensate for temporally autocorrelated deviations associated with deficiencies in the model. Several criteria need to be assessed to set the appropriate value of $\beta$. Obviously the

TABLE 4. Symbols and definitions of components from Tables 1 and 2.

\begin{tabular}{ll}
\hline \hline \multicolumn{1}{c}{ Symbol } & \multicolumn{1}{c}{ Description } \\
\hline $\mathbf{x}$ & model state vector \\
$f$ & model for projecting $\mathbf{x}$ forward in time \\
$\mathbf{u}$ & external model drivers \\
$\mathbf{d}$ & deviations of the model state vector from the ensemble mean state vector \\
$n$ & the number of ensemble members \\
$\mathbf{P}$ & covariance matrix for the ensemble of state vectors \\
$\mathbf{Z}$ & observation vector \\
$\mathbf{H}$ & observation matrix \\
$\mathbf{y}$ & innovations (deviations of the model predictions from observations) \\
$\mathbf{S}$ & covariance of model deviations assuming an unbiased model \\
$\mathbf{K}$ & Kalman correction matrix \\
$\mathbf{Q}$ & diagonal variance matrix of current error for the model \\
$\mathbf{\Psi}$ & diagonal variance matrix of current measurement errors \\
$N(\cdot)$ & noise generator (assumed zero-mean Gaussian) \\
Subscript $i$ & ensemble member \\
Subscript $t$ & time \\
Subscript $(t 1 \mid t 2)$ & indicates estimate at time $t 1$ with data assimilated up through time $t 2$ \\
Subscript $*$ & indicates state or values uncorrupted by $N(\mathbf{Q} t)$ \\
$T$ & indicates the transpose \\
$\mathbf{\Gamma}$ & error distribution matrix \\
$\beta$ & error weighting on observed variables \\
$\mathbf{I}$ & identity matrix \\
$\mathbf{Q}$ & inferred model error variance (only main diagonal element are retained) \\
$\alpha$ & weighting on prior estimates of $\mathbf{Q}$ for time smoothing, others as in Table 1 \\
\hline
\end{tabular}


output of the filter should follow the observations closely. However, if the filter output follows the observations too closely, then the random noise in the observations will have simply passed through the filter, which is at odds with the signal processing objective; thus, minimizing the squared or absolute deviations will not work. So how close is close enough? Three criteria can be used to address this question.

1) The first is to examine the variance among the ensemble of simulations relative to the variance of the observations. Clearly, there is no justification for the ensemble variance being smaller than that of the observations, nor is it desirable that it be much larger. Averaged over the entire time series, the ensemble variance in modeled net ecosystem carbon exchange (NEE; $\sigma_{\mathrm{NEE}}^{2}$, the main diagonal element of $\mathbf{P}_{t}$ associated with NEE) is about equal to the variance in the observations $\left(\sigma_{\text {OBS }}^{2}\right.$ ) when $\beta$ is $\sim 0.5$ (Fig. 4). For $\beta$ larger than this value, the ensemble variance increases exponentially.

2) The second criterion for setting the value of $\beta$ is based on the autocorrelation in the residuals between the ensemble mean and the observations (the mean of the innovations). In our data there is a distinct diel pattern in the mean of the innovations. This diel pattern is not caused by the cyclic variation in observation errors $\left(\boldsymbol{\Psi}_{t}\right)$ imposed by Eq. 3 (i.e., the diel pattern persists even when $\boldsymbol{\Psi}_{t}$ is held constant; data not shown). The diel pattern decreases as $\beta$ decreases and can be virtually removed with a $\beta$ of 0.1 (Fig. 5). However, the decrease in the diel pattern of residuals comes at the expense of wildly fluctuating leaf-area estimates (Fig. 6). With $\beta$ values of between 0.5 and 0.6 , a substantial decrease in the amplitude of the residuals can be achieved while maintaining a coherent leaf-area time series that is nonnegative.

3) The third criterion to consider when setting $\beta$ is the propagation of error from previous time steps. If $\beta$ is set too high or too low, then the noise added either to the NEE estimates $\left(q_{\mathrm{NEE}}^{2}\right.$, the diagonal element of the $\mathbf{Q}$ matrix associated with NEE) or to the LAI estimates $\left(q_{\mathrm{LAI}}^{2}\right.$, the diagonal element of the $\mathbf{Q}$ matrix associated with LAI) becomes very large (Fig. 4). This added noise propagates through to future estimates even after the correction step in the EnKF. The propagated error $\left(\sigma_{* \mathrm{NEE}}^{2}\right.$, the diagonal element of the $\mathbf{P} *_{t}$ matrix associated with NEE) is minimized at a $\beta$ value of $\sim 0.55$ (Fig. 4 ). This minimum in the propagated error arises because of the nonlinear tradeoffs between $q_{\mathrm{NEE}}$ and $q_{\mathrm{LAI}}$ as $\beta$ varies. At low values of $\beta$, increases in $\beta$ result in large reductions in noise imposed on LAI with minimal increases in the noise imposed on NEE (Fig. 4). At high values of $\beta$, decreases in $\beta$ result in large reductions in noise imposed on NEE with minimal increases in the noise imposed on LAI. The balance in this trade-off is at about $\beta=0.55$.

Based on all these criteria, we set $\beta$ to 0.55 for our analysis of the PLIRTLE model in relation to the eddy

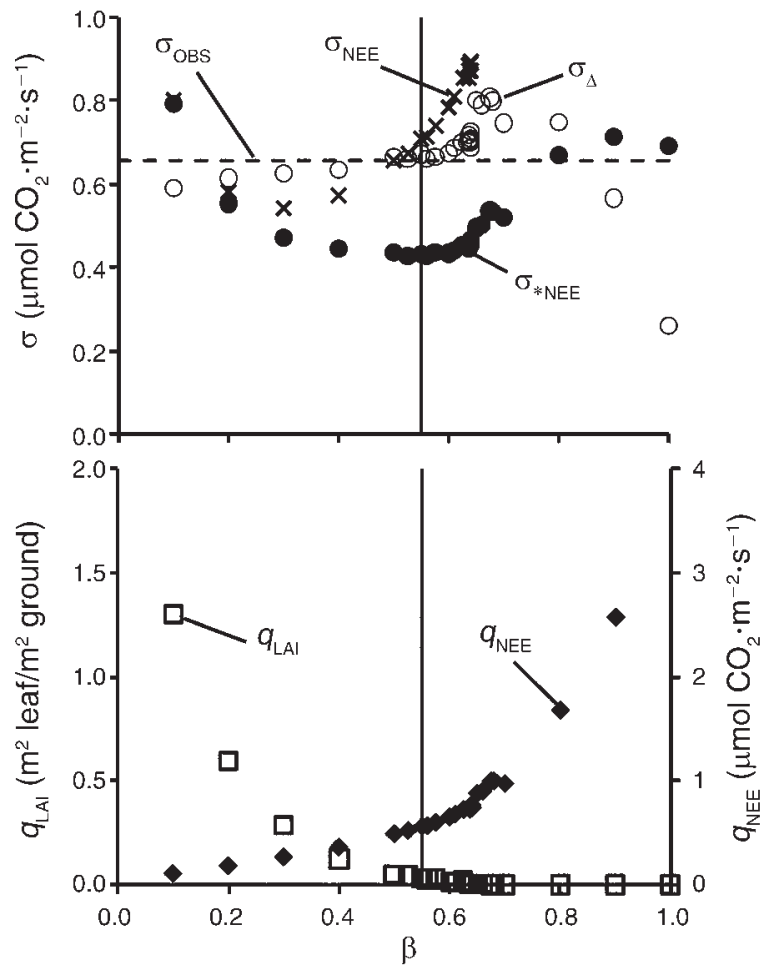

FIG. 4. Comparison of variances in the EnKF at various values of the error-distribution weighting factor $\beta$. The variances have been averaged over a two-week time series of half-hourly eddy covariance data and the associated standard deviations (SD) plotted here: $\sigma_{\mathrm{OBS}}$ is the SD of the NEE observations; $\sigma_{\Delta}$ is the SD of the mean EnKF output minus the observations; $\sigma_{\mathrm{NEE}}$ is the SD of the NEE estimates from the ensemble of model predictions (the square root of diagonal element of $\mathbf{P}_{t}$ associated with NEE); $\sigma_{* \mathrm{NEE}}$ is the SD of NEE estimates propagated forward by the ensemble of models from previous time steps (the square root of diagonal element of $\mathbf{P}_{* t}$ associated with NEE); $q_{\mathrm{NEE}}$ and $q_{\mathrm{LAI}}$ are the square roots of the elements in $\mathbf{Q}_{t}$ associated with NEE and LAI, respectively; they are the SD of the noise used to corrupt the model predictions to account for uncertainty in the model.

covariance data. Again, this value of $\beta$ does not minimize variance of residuals between the ensemble mean and the observations $\left(\sigma_{\Delta}\right.$; Fig. 4$)$. With either very low or very high values of $\beta$, the deviations of the observations from the mean of the corrected ensemble can be made substantially smaller (especially for large $\beta)$. However, this decrease in deviations is actually an indication of poor signal processing. With $\beta$ either small or large, the noise imposed on LAI or NEE, respectively, is also large. This large noise corrupts the model predictions sufficiently for the filter to shift the weighting in the correction step away from the model and toward the observations. Thus, with this large model uncertainty the filter ignores the model prediction and hence does not filter random noise out of the observations, which, of course, is contrary to the signal processing objective. 

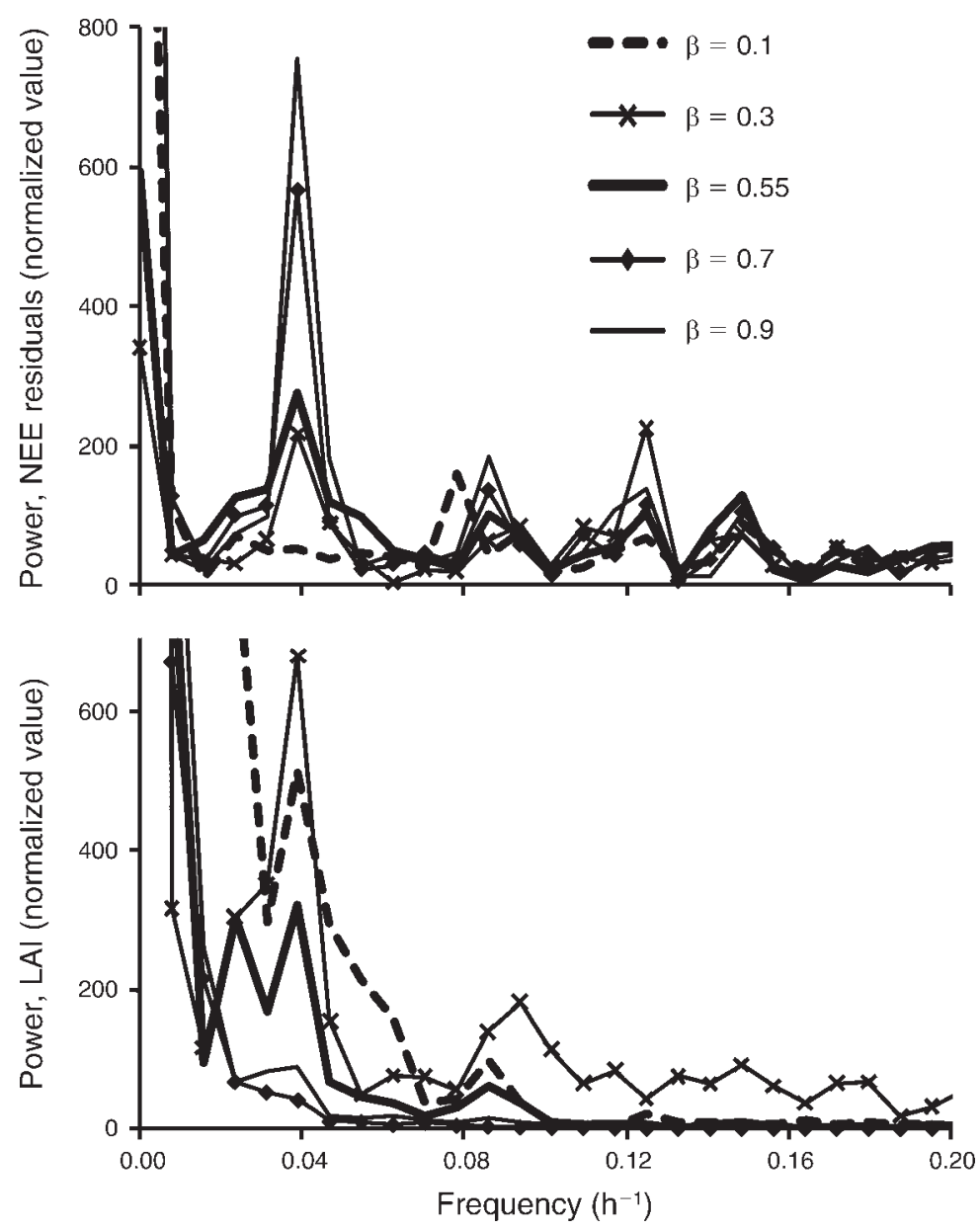

FIG. 5. Power spectra for the NEE residuals (mean EnKF estimate minus measured NEE) and LAI estimates from the EnKF at various values of the error-distribution weighting factor $\beta$. The strong peaks near a frequency of $0.04 \mathrm{~h}^{-1}$ indicate a strong $24-\mathrm{h}$ cycle in the NEE residuals and LAI estimates.

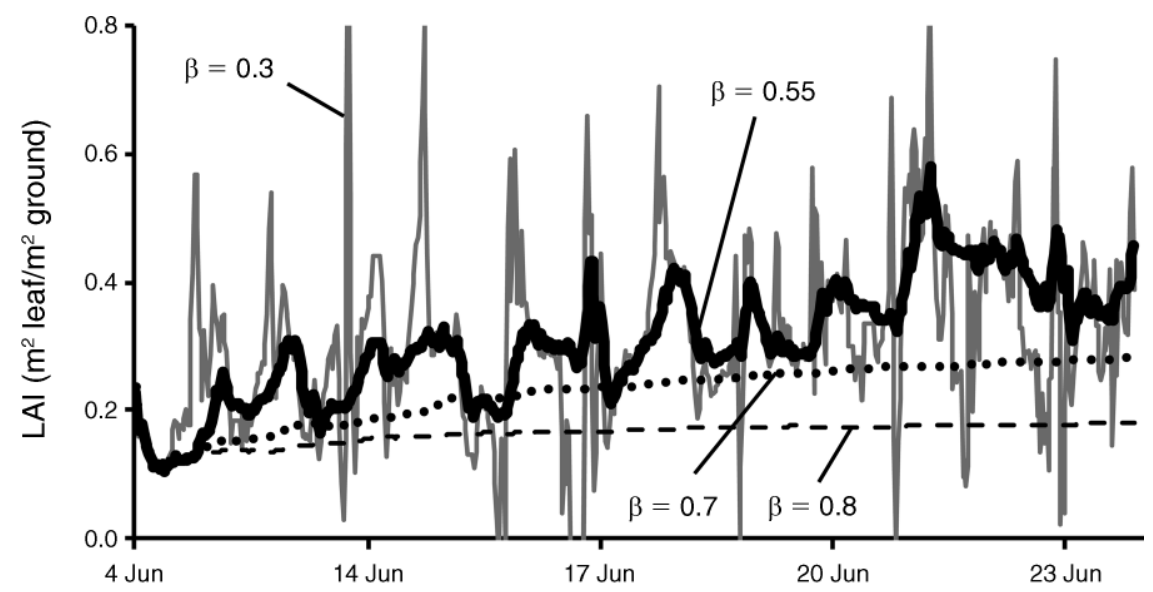

FIG. 6. EnKF estimates of leaf area with four values of the error-distribution weighting factor $\beta$. With $\beta$ small, most of the variation in the innovations gets distributed to leaf area and the leaf area estimates fluctuate wildly. With $\beta$ large, very little of the variation in the innovations is distributed to leaf area, and there is not enough variation in the ensemble of simulations to allow the estimates of leaf area to adapt to changes associated with canopy phenology, measured during June 2004. Our analysis indicates an optimum value for $\beta$ of $\sim 0.55$. 


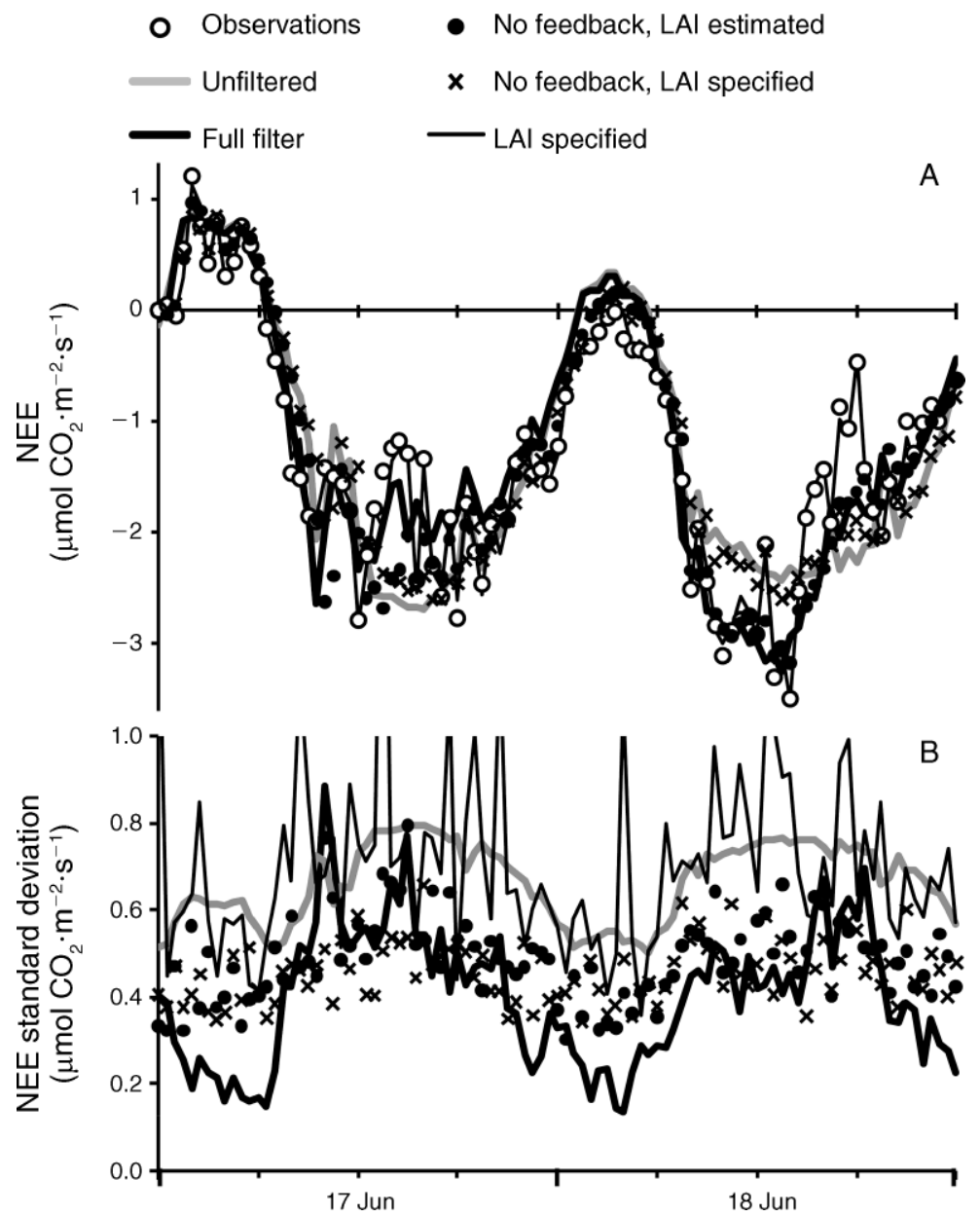

FIG. 7. Performance of the EnKF in four configurations: full filter with LAI added to the Kalman state vector to compensate for model deficiencies and with adaptive noise estimation to allow feedback from the filter deviations onto the Monte-Carlo noise added to the simulations; no feedback, LAI estimated, with LAI added to the Kalman state vector but without the adaptive noise estimation feedback; no feedback, LAI specified, without LAI added to the Kalman state vector and without the adaptive noise estimation feedback; LAI specified, without LAI added to the Kalman state vector but with the adaptive noise estimation feedback. Also shown are the PLIRTLE model predictions independent of the EnKF (unfiltered) and the observations. Standard deviations for the unfiltered model were calculated using Eq. 3. Estimates of (A) NEE and (B) its associated standard deviation are shown for the PLITRTLE model without the EnKF (unfiltered) and with the EnKF in four configurations, 17 and 18 June 2004. In panel B the peaks have been deliberately cut off to increase clarity of the figure.

\section{Compensating for autocorrelated residuals and adaptive noise estimation}

To assess the effects of sequential recalibration of LAI $(L)$ to account for model deficiencies and of adaptive noise estimation, we ran the PLIRTLE model, unfiltered, outside the EnKF and with various configurations of the EnKF (Fig. 7). To apply PLIRTLE outside the EnKF we had to assume a leaf-area phenology. We fit our phenology model for $L$ (Eq. 2) to estimates from the full EnKF with $\alpha=0.5$ and $\beta=0.55$ (Fig. 8C)

$$
\hat{L}_{t}=0.178+2.84 \times 10^{-5} \sum_{i=0}^{t} T_{i}
$$

where $\hat{L}_{t}$ is the modeled estimate of $L, t$ is time in halfhour increments since the beginning of the time series, and $T_{i}$ is the temperature at time $i$. Eq. 4 is simply the integrated form of Eq. 2 with $a=2.84 \times 10^{-5}$ and 0.178 as the initial LAI. Extrapolating this model forward using temperature records through July, LAI reaches a value of $\sim 0.7$ by the end of the first week in July, which is consistent with the values Williams et al. (2006) report for this same location and are consistent with the timing of leaf expansion for the North Slope of Alaska (Williams et al. 2001). By fitting this phenology model to the LAI estimates from the full EnKF, our unfiltered application of PLIRTLE includes the long-term phenological trend in LAI derived from the EnKF results, but does not include the higher-frequency daily adaptation of $L$ nor the a posteriori corrections based on an assessment of model vs. measurement error. 

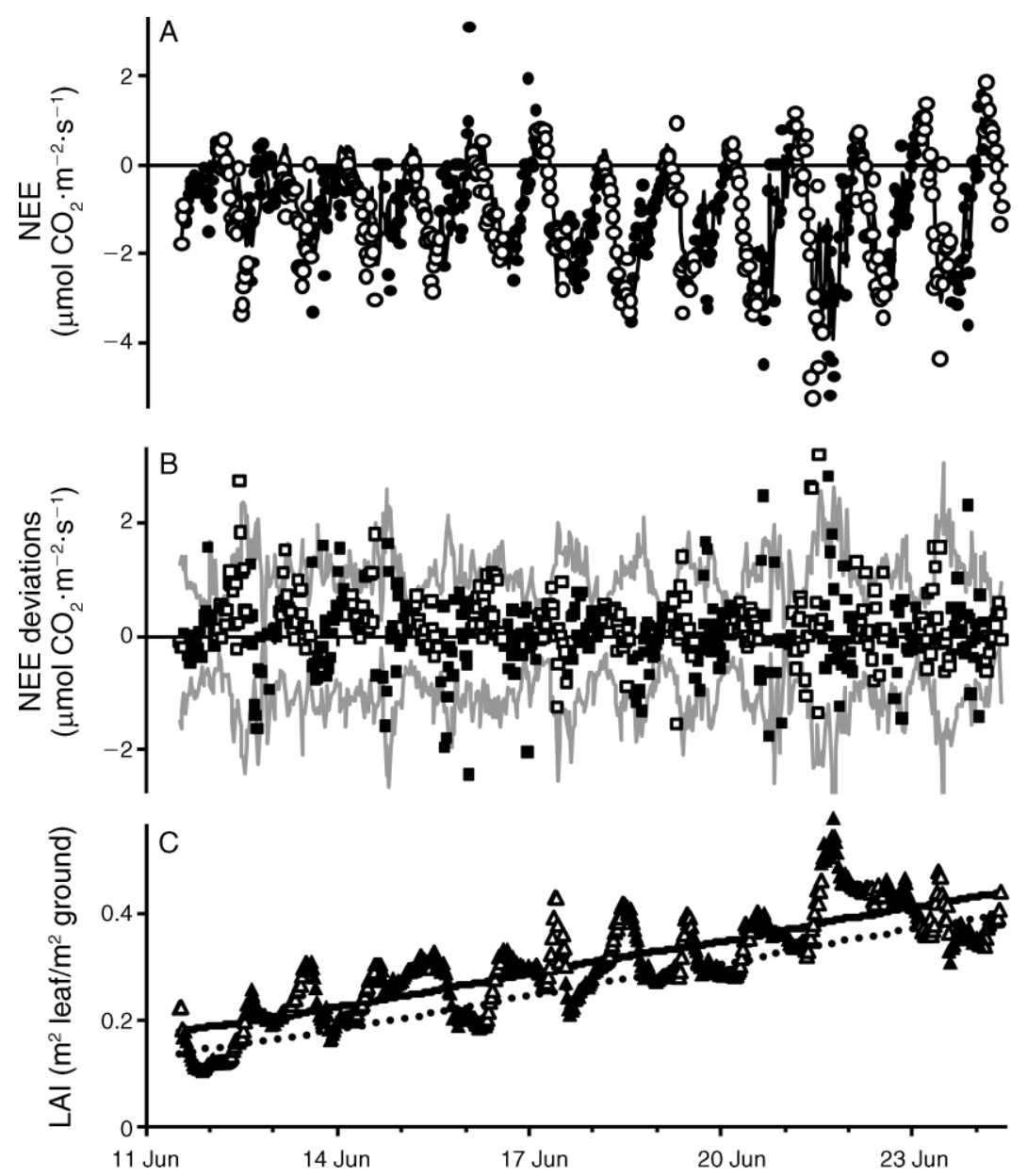

FIG. 8. Performance of the EnKF for a two-week time series in June 2004 of eddy covariance data from the North Slope of Alaska. Open symbols are for data collected between solar midnight and solar noon (a.m.); closed symbols are for data collected between solar noon and solar midnight (p.m.). Panel (A) shows the eddy covariance data (circles) and the mean of an ensemble of 100 simulations in the EnKF (black line); panel (B) shows the ensemble mean minus the observation (squares) and the range of the deviations of the ensemble of simulations from the ensemble mean (gray lines); panel (C) shows the EnKF estimates of effective leaf area (triangles), a temperature-sum model fit to these estimates (thick line; Eq. 4), and an offset from this model adjusted so that the mean $P_{\max }$ for separately fit a.m. and p.m. models equaled the Shaver et al. (2007) estimate (dotted line).

We also ran the model embedded in the EnKF in four configurations: (1) full filter, the EnKF as described with $\alpha=0.5$ and $\beta=0.55$; (2) without feedback, the EnKF without the feedback of adaptive noise estimation $(\alpha=$ $1)$ and with $q_{\mathrm{NEE}}$ and $q_{\mathrm{LAI}}$ set to their mean values from the full filter $\left(q_{\mathrm{NEE}}^{2}=0.316, q_{\mathrm{LAI}}^{2}=0.000963\right)$; (3) without feedback or compensation for autocorrelated deviations, the EnKF without feedback $(\alpha=1)$, with LAI as an input based on Eq. 4, with $q_{\mathrm{NEE}}$ set to its mean value from the full filter, but with no noise added to $L$ in the Kalman state vector; (4) without compensation for autocorrelated deviations, the EnKF with feedback $(\alpha=$ 0.5 ), with LAI as an input based on Eq. 4, but with no noise added to $L$ in the Kalman state vector.

We also calculated standard deviations for each NEE prediction in the time series for each of these applications (Fig. 7B). We assumed the standard deviations for the unfiltered model equaled those for the observations
(Eq. 3 with the unfiltered prediction used for $F_{\mathrm{C}}$ ). For the filtered data, we assumed that the $99 \%$ confidence limit was equal to the range of the 100 simulations in the Monte Carlo ensemble. One standard deviation was this range divided by 5.15 (the $99 \%$ confidence interval for a normal distribution with a standard deviation of one).

Even without the EnKF, the PLIRTLE model fits the data remarkably well (Fig. 7A). Over the full two weeks of data, the standard deviation of the PLIRTLE model from the observations is $0.74 \mu \mathrm{mol} \mathrm{CO} \mathrm{CO}_{2} \cdot \mathrm{m}^{-2} \cdot \mathrm{s}^{-1}(\sim 21 \%$ of the diel range in NEE). With the full filter, the standard deviation improved to $0.59 \mu \mathrm{mol} \cdot \mathrm{m}^{-2} \cdot \mathrm{s}^{-1}$ $(\sim 17 \%$ of the diel range in NEE).

\section{Full filter}

With the full filter $(\alpha=0.5, \beta=0.55, L$ estimated by the EnKF), the predictions track the autocorrelated pattern in the observations more closely than the 
unfiltered model, but effectively remove random spikes in the observations. Spike detection and removal is a critical component of processing eddy covariance data (Papale et al. 2006). Part of this improvement is trivially associated with the correction step in the EnKF, but a large part is associated with the compensation for model deficiencies through the sequential recalibration of $L$.

\section{Without feedback}

Without the feedback provided by the adaptive noise estimation $(\alpha=1, \beta$ ignored, $L$ estimated within the EnKF), the filter does nearly as well as the full filter for much of the time series, but takes a longer time to converge in response to autocorrelated deviations (e.g., Fig. 7 near noon on 17 June 2004). In this application, the noise levels added to NEE and LAI $\left(q_{\mathrm{NEE}}\right.$ and $\left.q_{\mathrm{LAI}}\right)$ were set to the mean levels from the full filter. This constant noise level still allows adjustments to LAI, but the rate of adjustment does not increase with persistently large innovations, as it does when $\alpha=0.5$. Occasionally this constant noise level is not large enough to allow the corrections to NEE and LAI needed to keep up with the observed NEE (e.g., near noon on 17 June).

\section{Without feedback or compensation for autocorrelated deviations}

Without the feedback and without compensating for autocorrelated deviations ( $\alpha=1, \beta$ ignored, $L$ specified with Eq. 4), the filter follows the predictions of the unfiltered model with small corrections based on a fixed noise level. Without the sequential recalibration of LAI, there is no propagation of information from one time step to the next. Therefore, there is no way for the filter to adjust to persistent deviations from the observations. Tracking of the data could be improved by increasing the noise level added to NEE $\left(q_{\mathrm{NEE}}\right)$, but this solution would also allow higher levels of data noise to pass through the filter.

\section{Without compensation for autocorrelated deviations}

Without compensating for autocorrelated deviations but allowing feedback through the adaptive noise estimation ( $\alpha=0.5, \beta=1, L$ specified with Eq. 4), allows the EnKF to track the observations very closely. However, this close tracking comes at the expense of allowing nearly all the data noise to pass through the filter. This application of the EnKF without compensating for autocorrelated deviations is equivalent to setting $\beta$ to 1 (see Fig. 4), but accounting for the phenology in LAI through an external input to the model. With $\beta$ set to one, there is only feedback to the noise level added to $\operatorname{NEE}\left(q_{\mathrm{NEE}}\right)$ and that noise level is very high. Thus the EnKF calculates a high estimate covariance $\left(\mathbf{P}_{t}\right)$ and therefore a high Kalman gain $\left(\mathbf{K}_{t}\right)$. With a high Kalman gain, the EnKF imposes a large correction on the predictions, which collapses the ensemble close to the observations and passes nearly all the data noise through the filter.
Over the two-week period we analyzed, the cumulative carbon flux into the ecosystem also differed among the unfiltered observations, the model outside of the filter, and the various applications of the filter. The unfiltered observations yielded the highest estimate of the cumulative flux into the ecosystem $\left(14.8 \mathrm{~g} \mathrm{C} / \mathrm{m}^{2}\right)$, suggesting large negative noise spikes (i.e., apparent carbon sinks) for most of the NEE time series (Fig. 8; e.g., 12 and 22 June 2004; but clearly not for the two days selected for Fig. 7 [17 and 18 June 2004]). The next highest cumulative flux into the ecosystem was for the EnKF without compensating for autocorrelated deviations, but with the adaptive noise estimation (14.3 g C/ $\mathrm{m}^{2}$ ); this application of the EnKF amplified the noise added to the model predictions and therefore passed most of the spikes in the observations through the filter. In the application without the feedback provided by adaptive noise estimation, but compensating for autocorrelated deviations, the flux estimate was still high $\left(13.7 \mathrm{~g} \mathrm{C} / \mathrm{m}^{2}\right)$; this application of the EnKF was slow to respond to persistent model-data deviations, suggesting that the model tended to overestimate photosynthesis relative to the observations during these persistent deviations (modeled NEE too negative). In the application with both our modifications, the cumulative flux estimate is only slightly higher $\left(12.8 \mathrm{~g} \mathrm{C} / \mathrm{m}^{2}\right)$ than with neither modification $\left(12.5 \mathrm{~g} \mathrm{C} / \mathrm{m}^{2}\right)$. This close agreement is probably a fortuitous cancelation of errors in the estimates by the EnKF with neither modification; with neither modification, NEE estimates tended to be high on some days but low on other days (Fig. 7). The lowest cumulative flux estimate was for the unfiltered PLIRTLE model outside of the EnKF (12.06 g C/m²); the unfiltered model tended to follow the same general trajectory as the EnKF with neither modification and therefore was also subject to the same error cancelation. In addition, this unfiltered estimate is very sensitive to the estimates of LAI we used to drive the model.

Distinction among the various applications of the EnKF can also be seen in the standard deviations of the ensemble of predictions (Fig. 7 bottom). The standard deviation of the observations increases and decreases with the magnitude of NEE (Eq. 3). This oscillation in the standard deviations passes through the EnKF because it is used to calculate the Kalman gain and hence to correct the predictions. Thus the standard deviations of the various configurations of the EnKF also oscillate on a daily basis. The standard deviation for the full filter adjusts as needed to correct for persistently large innovations. It can be small relative to the observation standard deviations (i.e., the model increases confidence in the data after assimilation), but increases as needed. Without the feedback of the adaptive noise estimation, the noise component of the standard deviations is constant (constant $\boldsymbol{q}_{\mathrm{NEE}}$ ) and the daily oscillation is associated strictly with the propagation of error in the estimates of LAI and the observation error passing through the filter. Without the feedback 
and without compensating for autocorrelated deviations, only the constant NEE noise and the observation noise contribute to the standard deviation. With feedback but without compensating for autocorrelated deviations, the EnKF adjusts the standard deviations of NEE to about the same level as those of the observations, but with occasional spikes associated with large deviations in the observations.

\section{Test of the PLIRTLE model}

As already pointed out, the PLIRTLE model fits the eddy covariance time series well even without the aid of the EnKF (Fig. 7, unfiltered data). However, there are important, nonrandom deviations of the model from the observations. In this section, we use the EnKF to analyze those deviations and identify research paths that might be pursued to improve the model.

Both the deviations of the NEE estimates from the observations and the estimates of LAI have a distinct diel pattern (Figs. 5, 6, and 8). These diel patterns are a clear indication that there is a deficiency in PLIRTLE that precludes it from capturing all of the nonrandom pattern in the NEE time series (Beck and Young 1976, Lin and Beck 2007). Even if we had adjusted $\beta$ so that the LAI estimates lost the diel pattern (i.e., increased $\beta$ $>0.7$, Fig. 6), the model would have still failed because the pattern transferred to LAI by the EnKF would simply have remained in the deviations (Fig. 5). The model failure is subtle (Fig. 7), but nevertheless nonrandom and therefore potentially significant. The failure is analogous to a regression analysis in which the residuals retain nonrandom pattern; the $r^{2}$ might be very high and the standard deviation of the residuals very small, but if the residuals still have a nonrandom pattern, the regression model has missed something important.

The EnKF estimates of NEE (Fig. 8A) tend to be high relative to the observations between solar midnight ( 2:00 a.m. Alaska Daylight Time) and solar noon (termed "a.m."), especially early in the time series (Fig. 8 open symbols). Between solar noon and solar midnight (termed "p.m.") the estimates are substantially less biased. Although the a.m. deviations are not significantly $>0$ over the time series $(0.27 \pm 0.57 \mu \mathrm{mol}$ $\mathrm{CO}_{2} \cdot \mathrm{m}^{-2} \cdot \mathrm{s}^{-1}$ ), they are sufficient to drive the LAI estimates upward in the morning of most days (Fig. 8). The LAI estimates decline on most afternoons. The amplitude of the daily excursions of LAI estimates from the long-term trend (Eq. 4) was $\pm 0.16 \mathrm{~m}^{2} / \mathrm{m}^{2}$ (standard deviation of LAI estimates after subtracting Eq. 4). Based on 10 passes of the data through the EnKF (with independent ensembles and noise corruption), the standard deviation for any single estimate of LAI at a particular time was an order of magnitude smaller $\left( \pm 0.015 \mathrm{~m}^{2} / \mathrm{m}^{2}\right)$. Thus, the diel pattern in LAI is highly significant $(P<0.001)$. With 24 hours of sunlight, the slope of the photosynthesis $(P)$ equation with respect to leaf area is almost always larger than the slope of the respiration $(R)$ equation with respect to leaf area $(L ; d P /$ $d L>d R / d L$ in Eq. 1). Thus, the pattern of a.m. increasing LAI and p.m. declining LAI suggests an a.m. underestimate of photosynthesis by the model relative to respiration and a p.m. overestimate of photosynthesis relative to respiration.

This conclusion is supported by independent estimates of a.m. vs. p.m. values for $P_{\max }$ and $E_{0}$ (quantum yield; leaving the remaining four PLIRTLE parameters fixed; Table 3). We assumed LAI based on Eq. 4, but adjusted it downward until the mean of a.m. and p.m. estimates of $P_{\max }$ equaled the value reported by Shaver et al. (2007). This new estimate of the LAI phenology passes near the troughs in the EnKF estimates of LAI (dotted line in Fig. 8C bottom). We then corrupted the observed NEE data with Gaussian noise using the variance from Eq. 3 and repeated the fit 10 times. Based on these fits, the mean a.m. $P_{\max }$ was significantly higher than the p.m. value $(17.36 \pm 0.46$ vs. $14.02 \pm 0.31 \mu \mathrm{mol}$ $\left.\mathrm{CO}_{2} \cdot \mathrm{m}^{-2} \cdot \mathrm{s}^{-1} ; t=18.99, P<0.001\right)$, but the a.m. and p.m. values of $E_{0}$ did not differ significantly $(0.0549 \pm$ 0.003 vs. $0.0551 \pm 0.003 \mu \mathrm{mol} \mathrm{CO} / \mu \mathrm{mol}$ photons; $t=$ $0.18, P>0.1)$. These values of $E_{0}$ are substantially higher than those reported by Shaver et al. (2007) based on their fit to chamber-based NEE $\left(0.036 \mu \mathrm{mol} \mathrm{CO}_{2} /\right.$ $\mu$ mol photons), but lower than the values they reported for their photosynthesis equation fit to just photosynthesis data $(0.069 \mu \mathrm{mol} \mathrm{CO} / / \mu \mathrm{mol}$ photons; Shaver et al. 2007: Table 5).

There are three obvious candidate causes for the unexpected diel excursions in the NEE and LAI estimates.

1) There is a potential bias associated with heating and cooling of the open-path infrared gas analyzer (Burba et al. 2008). However, the deviations in our analysis do not have the symmetry around solar noon seen in the Burba et al. (2008) analysis and the diel pattern of deviations persists even for closed-path sensors we have analyzed at other locations in the Arctic (proprietary data not shown).

2) There is a potential bias associated with changes in wind speed and direction, which affect the location of the area being sampled around the tower (tower footprint); the diel pattern might therefore reflect spatial heterogeneity in the biological and physical characteristics of the tower footprint. However, we see the same morning underestimate and afternoon overestimate of the $\mathrm{CO}_{2}$ sink at the other arctic sites we have analyzed; it is unlikely that all sites would have the same spatial arrangement of vegetation around the tower.

3) Finally, low afternoon photosynthesis is potentially caused by stomatal closure driven by low humidity (Ball et al. 1987, Leuning et al. 1995). Gebauer et al. (1998) reported afternoon stomatal closure in response to high vapor pressure deficit (VPD) for the tussock-forming sedges that dominate the footprint of our tower (Eriophorum vaginatum). This stomatal closure occurred even under moist soil conditions. To examine this 


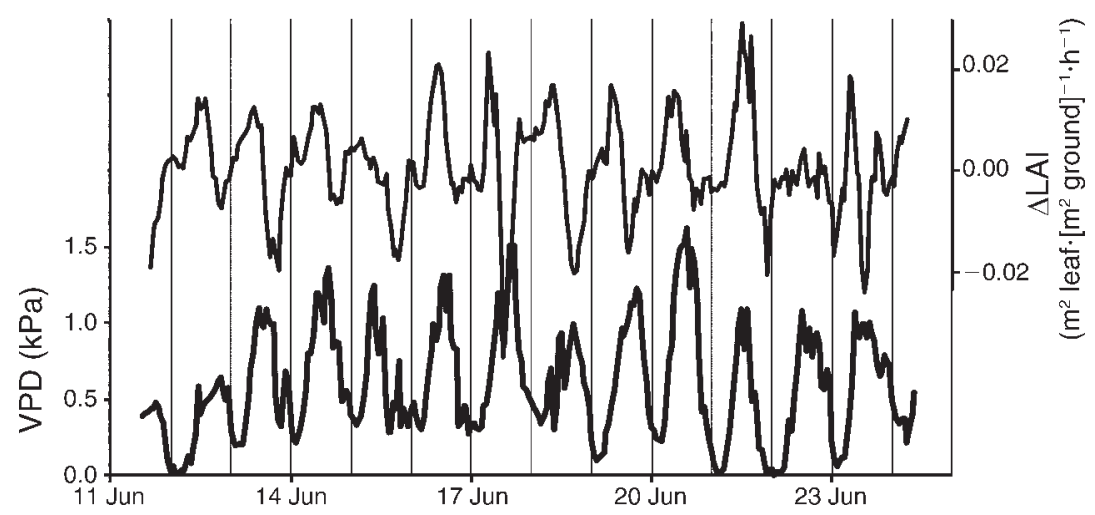

FIG. 9. Correspondence between adaptation rate of LAI estimates from the EnKF $\left(\Delta \mathrm{LAI}=L_{t+1}-L_{t}\right)$ and vapor pressure deficit (VPD) in June 2004. The LAI adaptation rate has been smoothed with a six-hour running mean.

possibility, we compared the adaptation rate of LAI estimates from the EnKF with estimates of VPD (Fig. 9). The adaptation rate was calculated as the difference between consecutive estimates of LAI, then smoothed with a six-hour running mean. There is a fair correspondence between the peaks in VPD and declining LAI estimates $\left(r^{2}=0.13, n=303, P<0.01\right)$. Although the LAI-VPD correlation is clearly not conclusive, it is suggestive and deserves further study.

The three potential causes for the diel pattern would not likely have been detected by Shaver et al. (2007) using a chamber-based estimate of NEE. The chamberbased method is not sensitive to warming and cooling the way that open-path sensors are (Burba et al. 2008), changes in wind direction obviously have no effect on the fluxes within a sealed $1-\mathrm{m}^{2}$ chamber, and humidity would be expected to increase in the chambers, thereby hiding any VPD effect on stomatal opening. If the first potential cause underlies the pattern we detected, then the pattern is probably particular to only eddy covariance data. If the second potential cause underlies the pattern, then it has real-world relevance only in terms of spatial patterns of NEE. If the third potential cause underlies the pattern, then the PLIRTLE model is clearly deficient in describing the complete pattern in NEE for individual patches of tundra. Distinguishing among potential causes for the diel pattern in the EnKF LAI estimates in future studies will advance the understanding of arctic $\mathrm{C}$ dynamics and will improve the ability to model these systems and predict changes in C flux over time

\section{Summary And Conclusions}

We presented two modifications to the ensemble Kalman filter (EnKF) that improve its performance for filtering time-series data. The first modification was to compensate for autocorrelated deviations between predictions and observations by allowing the EnKF to sequentially recalibrate unobserved components of the Kalman state vector (Lin and Beck 2007). The unobserved components of the state vector can either be variables in the embedded model or parameters augmented to the state vector. In either case, the recalibrated variables must have a direct effect on the observed variables through the model structure and must themselves be autocorrelated so that the recalibration is propagated in time.

The second modification was to add an adaptive noise estimation algorithm to the EnKF (Jazwinski 1998). Estimating the noise associated with the model structure is one of the most difficult aspects of applying the Kalman filter. The adaptive noise estimation automates this process and allows the filter to converge quickly when there are persistent deviations between model and observation and to narrow confidence limits when the model is tracking the data well.

We embedded a model of arctic net ecosystem carbon exchange (NEE; the PLIRTLE model; Shaver et al. 2007) into the EnKF and applied it to eddy covariance data for a site on the North Slope of Alaska. The model tracked the data well even without the EnKF. However, the EnKF effectively filtered the NEE estimates both by removing random noise in the eddy covariance data and by compensating for autocorrelated deviations associated with deficiencies in the model.

Without either of our modifications to the EnKF, the filter output followed unfiltered model predictions closely. Data tracking for the unmodified EnKF could be improved by increasing the noise levels associated with the model structure, but only at the expense of passing more noise through the filter. Accounting for autocorrelated deviations in the EnKF, but without the adaptive noise estimation, improved the filter performance slightly over the unmodified EnKF, but the filter was slow to converge in response to persistent deviations between the data and model predictions. Again, the convergence behavior could be improved by increasing the model noise levels, but only at the expense of increasing uncertainty in the filter output. Adding the adaptive noise estimation without accounting for autocorrelated deviations resulted in very high levels of model noise. This high noise level allowed the filter to 
track the data nearly perfectly, and thus the EnKF did not filter out spikes in the data stream. The performance of the EnKF with only the adaptive noise estimation can be improved by increasing the autocorrelation of the noise estimates (increasing $\alpha$ ), but can never adequately account for autocorrelated deviations.

To apply the EnKF with both of our improvements, we had to adjust the distribution of model noise between observed and unobserved model components (set $\beta$ ). This adjustment determines how fast the unobserved components of the Kalman state vector can adapt and thus how the filter compensates for model deficiencies. If too much noise is distributed toward the observed variables, the filter passes a large fraction of the noise in the data and does not allow adaptation in the unobserved variables. If too much of the added noise is distributed to the unobserved variables, their estimates fluctuate wildly, also increasing noise in the filter output. We suggest these guidelines for adjusting the value of $\beta$ : (1) maintain the total noise added to the model predictions at a level near the measurement noise; (2) maintain coherence in the time-series estimates of the unobserved components of the Kalman state vector; and (3) minimize noise propagated forward from previous time steps by the ensemble of model predictions. Minimizing propagated uncertainty is particularly important in any model with autocorrelated dynamics, whether within the EnKF or not.

Finally we used the EnKF to test the PLIRTLE model of Shaver et al. (2007) of arctic carbon exchange. If the model embedded in the EnKF is an adequate description of the data being filtered, then sequential recalibration of the unobserved components of the Kalman state vector should result in only random variation in the estimates of these components about a fixed mean; any nonrandom variation in these estimates is an indication of a deficiency in the model. In our applications of the EnKF, we found two deficiencies in the PLIRTLE model.

First, leaf area index (LAI) estimates increased progressively through the two week simulations; this increase was anticipated and is consistent with springtime leaf emergence during the sampling period. The EnKF allowed us to assimilate NEE data into the PLIRTLE model to infer LAI through the sampling period.

Second, we also found a diel pattern in the LAI estimates that was clearly not random. This diel pattern in the LAI estimates resulted from fairly subtle deviations between the model predictions and the eddy covariance data. The pattern is indicative of a deficiency in the PLIRTLE structure in its representation of arctic NEE and its measurement with eddy covariance towers. We identified three potential causes for the diel pattern in our LAI estimates: (1) measurement bias associated with warming and cooling of the IRGA, (2) changes in the tower footprint associated with changing wind speed and direction, and (3) the omission of vapor pressure deficit (VPD) effects on stomatal opening in the PLIRTLE model.

\section{ACKNOWLEDGMENTS}

This material is based upon work supported by the U.S. National Science Foundation under grants OPP-0352897, DEB-0423385, DEB-0439620, DEB-0444592, and OPP0632139. The UK Natural Environment Research Council also funded part of this work through the CarbonFusion International Opportunities grant. Any opinions, findings, conclusions, or recommendations expressed in the material are those of the authors and do not necessarily reflect the views of the National Science Foundation or the Natural Environment Research Council.

\section{Literature Cited}

Anthoni, P. M., B. E. Law, and M. H. Unsworth. 1999. Carbon and water vapor exchange of an open-canopied ponderosa pine ecosystem. Agricultural and Forest Meteorology 95: $115-168$.

Baldocchi, D. D. 2003. Assessing the eddy covariance technique for evaluating carbon dioxide exchange rates of ecosystems: past, present, and future. Global Change Biology 9:479-492.

Ball, J. T., I. E. Woodrow, and J. A. Berry. 1987. A model predicting stomatal conductance and its contributions to the control of photosynthesis under different environmental conditions. Pages 221-224 in J. Biggins, editor. Progress in photosynthesis research. Volume IV. Martinus Nijhoff, Dordrecht, The Netherlands.

Beck, B., and P. Young. 1976. Systematic identification of DOBOD model structure. Journal of Environmental Engineering Division, ASCE 102:909-924.

Brown, A. G. 1983. Introduction to Random signal analysis and Kalman filtering. John Wiley and Sons, New York, New York, USA.

Burba, G. G., D. K. McDermitt, A. Grelle, D. J. Anderson, and L. Xu. 2008. Addressing the influence of instrument surface heat exchange on the measurement of $\mathrm{CO}_{2}$ flux from openpath analyzers. Global Change Biology 14:1854-1876.

Cava, D., U. Giostra, M. B. S. Siqueira, and G. G. Katul. 2004. Organized motion and radiative perturbations in the nocturnal canopy sublayer above an even-aged pine forest. Boundary Layer Meteorology 112:129-157.

Chen, M., S. Liu, L. L. Tieszen, and D. Y. Hollinger. 2008. An improved state-parameter analysis of ecosystem models using data assimilation. Ecological Modelling 219:317-326.

Cosby, B. J., and G. M. Hornberger. 1984. Identification of photosynthesis-light models for aquatic systems: I. Theory and simulations. Ecological Modelling 22:1-24.

Cosby, B. J., G. M. Hornberger, and M. G. Kelly. 1984. Identification of photosynthesis-light models for aquatic systems: II. Application to a macrophyte dominated stream. Ecological Modelling 22:25-51.

Ehman, J. L., H. P. Schmid, C. S. B. Grimmond, J. C. Randolph, P. J. Hanson, C. A. Wayson, and F. D. Cropley. 2002. An initial intercomparison of micrometeorological and ecological inventory estimates of carbon exchange in a midlatitude deciduous forest. Global Change Biology 8:575-589.

Evensen, G. 2003. The ensemble Kalman filter: theoretical formulation and practical implementation. Ocean Dynamics 53:343-367.

Falge, E., et al. 2002a. Seasonality of ecosystem respiration and gross primary production as derived from fluxnet measurements. Agricultural and Forest Meteorology 113:53-74.

Falge, E., et al. 2002b. Phase and amplitude of ecosystem carbon release and uptake potentials as derived from FLUXNET measurements. Agricultural and Forest Meteorology 113:75-95.

Fox, A. M., B. Huntley, C. R. Lloyd, M. Williams, and R. Baxter. 2008. Net ecosystem exchange over heterogeneous 
Arctic tundra: scaling between chamber and eddy covariance measurements. Global Biogeochemical Cycles 22:GB2027.

Gebauer, R. L. E., J. F. Reynolds, and J. D. Tenhunen. 1998. Diurnal patterns of $\mathrm{CO}_{2}$ and $\mathrm{H}_{2} \mathrm{O}$ exchange of the arctic sedges Eriophorum angustifolium and E. vaginatum (Cyperaceae). American Journal of Botany 85:592-599.

Goulden, M. L., B. C. Daube, S.-M. Fan, D. J. Sutton, A. Bazzaz, J. W. Munger, and S. C. Wofsy. 1997. Physiological responses of a black spruce forest to weather. Journal of Geophysical Research 102:28987-28996.

Gove, J. H., and D. Y. Hollinger. 2006. Application of a dual unscented Kalman filter for simultaneous state and parameter estimation in problems of surface-atmosphere exchange. Journal of Geophysical Research 111:D08S07.

Hanan, N. P., G. Burba, S. B. Verma, J. A. Berry, A. Suyker, and E. A. Walter-Shea. 2002. Inversion of net ecosystem $\mathrm{CO}_{2}$ flux measurements for estimation of canopy PAR absorption. Global Change Biology 8:563-574.

Hollinger, D. Y., and A. D. Richardson. 2005. Uncertainty in eddy covariance measurements and its application to physiological models. Tree Physiology 25:873-885.

Jazwinski, A. H. 1998. Stochastic process and filtering theory. Dover Publications, Mineola, New York, USA.

Kane, D. L., and L. D. Hinzman. 2004. Climate data from the North Slope Hydrology Research project. University of Alaska Fairbanks, Water and Environmental Research Center. 〈http://www.uaf.edu/water/projects/NorthSlope〉

Law, B. E., et al. 2002. Environmental controls over carbon dioxide and water vapor exchange of terrestrial vegetation. Agricultural and Forest Meteorology 113:97-120.

Law, B. E., O. Sun, J. Campbell, S. Van Tuyl, and P. Thornton. 2003. Changes in carbon storage and fluxes in a chronosequence of ponderosa pine. Global Change Biology 9:510524.

Leuning, R., F. M. Kelliher, D. G. G. de Pury, and E. D. Schulze. 1995. Leaf nitrogen, photosynthesis, conductance and transpiration: scaling from leaves to canopies. Plant, Cell and the Environment 18:1183-1200.

Lin, Z., and M. B. Beck. 2007. Understanding complex environmental systems: a dual approach. Environmetrics 18:11-26.

Papale, D., M. Reichstein, M. Aubinet, E. Cafora, C. Bernhofer, W. Kutsch, B. Longdoz, S. Rambal, R. Valentini, T. Vesala, and D. Yakir. 2006. Towards a standardized processing of net ecosystem exchange measured with eddy covariance technique: algorithms and uncertainty estimation. Biogeosciences 3:571-583.

R Development Core Team. 2006. R: a language and environment for statistical computing. Version 2.3.1. R Foundation for Statistical Computing, Vienna, Austria.

Richardson, A. D., D. Y. Hollinger, G. G. Burba, K. J. Davis, L. B. Flanagan, G. G. Katul, J. W. Munger, D. M. Ricciuto, P. C. Stoy, A. E. Suyker, S. B. Verma, and S. C. Wofsy. 2006. A multi-site analysis of random error in tower-based measurements of carbon and energy fluxes. Agricultural and Forest Meteorology 136:1-18.

Running, S. W., D. D. Baldocchi, D. P. Turner, S. T. Gower, P. S. Bakwin, and K. A. Hibbard. 1999. A global terrestrial monitoring network integrating tower fluxes, flask sampling, ecosystem modeling and EOS satellite data. Remote Sensing of Environment 70:108-127.

Sacks, W. J., D. S. Schimel, R. K. Monson, and B. H. Braswell. 2006. Model-data synthesis of diurnal and seasonal $\mathrm{CO}_{2}$ fluxes at Niwot Ridge, Colorado. Global Change Biology 12: $240-259$

Shaver, G. R., L. E. Street, E. B. Rastetter, M. T. van Wijk, and M. Williams. 2007. Functional convergence in regulation of net $\mathrm{CO}_{2}$ flux in heterogeneous tundra landscapes in Alaska and Sweden. Journal of Ecology 95:802-817.

Turnipseed, A. A., D. E. Anderson, P. D. Blanken, W. M. Baugh, and R. K. Monson. 2003. Airflows and turbulent flux measurement in mountainous terrain part 1. Canopy and local effects. Agricultural and Forest Meteorology 119:1-21.

Vallino, J. J. 1985. On-line estimation of the oxygen-masstransfer coefficient and other state variables in a chemostat. Thesis. Department of Chemical Engineering California Institute of Technology, Pasadena, California, USA.

Wang, B., X. Zou, and J. Zhu. 2000. Data assimilation and its applications. Proceedings of the National Academy of Sciences USA 97:11143-11144.

Webb, E. K., G. I. Pearman, and R. Leuning. 1980. Correction of flux measurements for density effects due to heat and water vapor transfer. Quarterly Journal of the Royal Meteorological Society 106:85-100.

Wilczak, J. M., S. P. Oncley, and S. A. Stage. 2001. Sonic anemometer tilt correction algorithms. Boundary Layer Meteorology 99:127-150.

Williams, M., E. B. Rastetter, D. N. Fernandes, M. L. Goulden, S. C. Wofsy, G. R. Shaver, J. M. Melillo, J. W. Munger, S.-M. Fan, and K. J. Nadelhoffer. 1996. Modelling the soil-plant-atmosphere continuum in a Quercus-Acer stand at Harvard Forest: the regulation of stomatal conductance by light, nitrogen and soil/plant hydraulic properties. Plant, Cell and Environment 19:911-927.

Williams, M., E. B. Rastetter, G. R. Shaver, J. E. Hobbie, E. Carpino, and B. L. Kwiatkowski. 2001. Primary production of an arctic watershed: an uncertainty analysis. Ecological Applications 11:1800-1816.

Williams, M., P. A. Schwarz, B. E. Law, J. Irvine, and M. R. Kurpius. 2005. An improved analysis of forest carbon dynamics using data assimilation. Global Change Biology 11:89-105.

Williams, M., L. E. Street, M. T. van Wijk, and G. R. Shaver. 2006. Identifying differences in carbon exchange among arctic ecosystem types. Ecosystems 9:288-304.

Wofsy, S. C., M. L. Goulden, J. W. Munger, S.-M. Fan, P. S. Bakwin, B. C. Daube, S. L. Bassow, and F. A. Bazzaz. 1993. Net exchange of $\mathrm{CO}_{2}$ in a mid-latitude forest. Science 260: $1314-1317$.

Wu, X., Y. Luo, E. Weng, L. White, Y. Ma, and X. Zhou. 2009. Conditional inversion to estimate parameters from eddy-flux observations. Journal of Plant Ecology 2:55-68.

Young, P. 1984. Recursive estimation and time-series analysis: an introduction. Springer-Verlag Berlin, Germany. 